\title{
Pacific
}

Journal of

Mathematics

\section{RYSHKOV DOMAINS OF REDUCTIVE ALGEBRAIC GROUPS}

TAKAO WATANABE 


\title{
RYSHKOV DOMAINS OF REDUCTIVE ALGEBRAIC GROUPS
}

\author{
TAKAO WATANABE
}

Dedicated to Professor Ichiro Satake on his 85th birthday

\begin{abstract}
Let $G$ be a connected reductive algebraic group defined over a number field $k$. In this paper, we introduce the Ryshkov domain $R$ for the arithmetical minimum function $\mathrm{m}_{Q}$ defined from a height function associated to a maximal k-parabolic subgroup $Q$ of $G$. The domain $\mathrm{R}$ is a $Q(\mathrm{k})$-invariant subset of the adele group $G(\mathbb{A})$. We show that a fundamental domain $\Omega$ for $Q(k) \backslash$ R yields a fundamental domain for $G(k) \backslash G(\mathbb{A})$. We also see that any local maximum of $m_{Q}$ is attained on the boundary of $\Omega$.
\end{abstract}

\section{Introduction}

Let $\mathrm{P}_{n}$ be the cone of positive definite $n$ by $n$ real symmetric matrices, and let $\mathrm{m}(A)$ be the arithmetical minimum $\min _{0 \neq x \in \mathbb{Z}^{n}}{ }^{t} x A x$ of $A \in \mathrm{P}_{n}$. The function $f: A \mapsto \mathrm{m}(A) /(\operatorname{det} A)^{1 / n}$ on $\mathrm{P}_{n}$ is called the Hermite invariant. Since the maximum of $f$ gives the Hermite constant $\gamma_{n}$ for dimension $n$, the determination of local maxima of $f$ is a fundamental problem of lattice sphere packings in Euclidean spaces and the arithmetic theory of quadratic forms. Voronoi's theorem [1908, Théorème 17] states that $f$ attains a local maximum at a point $A$ if and only if $A$ is perfect and eutactic. Moreover, perfect forms play an essential role in Voronoi's reduction theory of $\mathrm{P}_{n}$ with respect to the action of $\mathrm{GL}_{n}(\mathbb{Z})$ (see, e.g., [Martinet 2003] and [Schürmann 2009]). Ryshkov [1970] introduced a locally finite polyhedron $\mathrm{R}(\mathrm{m})$ in $\mathrm{P}_{n}$ defined by the condition $\mathrm{m}(A) \geq 1$. It is not difficult to show that $A$ is perfect with $\mathrm{m}(A)=1$ if and only if $A$ is a vertex of the boundary of $\mathrm{R}(\mathrm{m})$. In particular, any local maximum of the Hermite invariant $f$ is attained on the boundary of $R(m)$. In this sense, we can say that the Ryshkov polyhedron $\mathrm{R}(\mathrm{m})$ is well matched with $f$.

Let $G$ be a connected isotropic reductive algebraic group defined over a number field k, and let $Q$ be a maximal k-parabolic subgroup of $G$. In previous papers [Watanabe 2000; 2003], we investigated a constant $\gamma(G, Q, \mathrm{k})$ as a generalization of Hermite's constant $\gamma_{n}$. Precisely, the constant $\gamma(G, Q, \mathrm{k})$ is defined to be

Keywords: reduction theory, fundamental domain, Hermite constant. 
the maximum of the function $\mathrm{m}_{Q}(g)=\min _{x \in Q(\mathrm{k}) \backslash G(\mathrm{k})} H_{Q}(x g)$ on $G(\mathrm{k}) \backslash G(\mathbb{A})^{1}$, where $H_{Q}$ denotes the height function associated to $Q$. To prove the existence of the maximum of $\mathrm{m}_{Q}$, we used Borel and Harish-Chandra's reduction theory for the adele group $G(\mathbb{A})$ with respect to $G(\mathrm{k})$. However, a Siegel set in $G(\mathbb{A})$ is not well matched with $\mathrm{m}_{Q}$ in a sense that one cannot obtain any information on locations of extreme points of $\mathrm{m}_{Q}$ in a Siegel set.

The purpose of this paper is to construct a fundamental domain of $G(\mathbb{A})^{1}$ with respect to $G(\mathrm{k})$ which is well matched with $\mathrm{m}_{Q}$. We first consider an analog of the Ryshkov polyhedron. We set $X_{Q}(g)=\left\{x \in Q(\mathrm{k}) \backslash G(\mathrm{k}): m_{Q}(g)=H_{Q}(x g)\right\}$ for a given $g \in G(\mathbb{A})^{1}$. This is a finite subset of $Q(\mathrm{k}) \backslash G(\mathrm{k})$ and is regarded as an analog of the set of minimal vectors of a positive definite real quadratic form. We define the domain $\mathrm{R}\left(\mathrm{m}_{Q}\right)$ as follows:

$$
\mathrm{R}\left(\mathrm{m}_{Q}\right)=\left\{g \in G(\mathbb{A})^{1}: \bar{e} \in X_{Q}(g)\right\},
$$

where $\bar{e}$ denotes the trivial class $Q(\mathrm{k})$ in $Q(\mathrm{k}) \backslash G(\mathrm{k})$. The set $\mathrm{R}\left(\mathrm{m}_{Q}\right)$ is a left $Q(\mathrm{k})$ invariant closed set with nonempty interior. The interior of $\mathrm{R}\left(\mathrm{m}_{Q}\right)$ is just a subset $\mathrm{R}_{1}$ consisting of $g \in \mathrm{R}\left(\mathrm{m}_{Q}\right)$ such that $X_{Q}(g)$ is the one-point set $\{\bar{e}\}$. We denote by $\mathrm{R}_{1}^{-}$the closure of $\mathrm{R}_{1}$ in $G(\mathbb{A})^{1}$. Both $\mathrm{R}_{1}$ and $\mathrm{R}_{1}^{-}$are also left $Q(\mathrm{k})$-invariant. By Baer and Levi's theorem [1931, Satz 7], there exists an open fundamental domain $\Omega_{Q}$ of $\mathrm{R}_{1}^{-}$with respect to $Q(\mathrm{k})$, that is, $\Omega_{Q}$ is a relatively open subset of $\mathrm{R}_{1}^{-}$ satisfying

- $Q(\mathrm{k}) \Omega_{Q}^{-}=\mathrm{R}_{1}^{-}$, where $\Omega_{Q}^{-}$denotes the closure of $\Omega_{Q}$ in $\mathrm{R}_{1}^{-}$, and

- $\gamma \Omega_{Q} \cap \Omega_{Q}^{-}=\varnothing$ for any $\gamma \in Q(\mathrm{k}) \backslash\{e\}$.

Let $\Omega_{Q}^{\circ}$ denote the interior of $\Omega_{Q}$ in $G(\mathbb{A})^{1}$. Then our main theorem is stated as follows:

Theorem. The set $\Omega_{Q}^{\circ}$ is an open fundamental domain of $G(\mathbb{A})^{1}$ with respect to $G(\mathrm{k})$. Any local maximum of $\mathrm{m}_{Q}$ is attained on the intersection of the boundary of $\Omega_{Q}^{\circ}$ and the boundary of $\mathrm{R}_{1}^{-}$.

If we denote by $r_{G}$ the k-rank of the commutator subgroup of $G$, then $G$ has $r_{G}$ standard maximal k-parabolic subgroups. Since $\Omega_{Q}$ depends on $Q$, we obtain $r_{G}$ different kinds of fundamental domains of $G(\mathbb{A})^{1}$ with respect to $G(\mathrm{k})$. The method to construct $\Omega_{Q}$ may be viewed as a generalization of the highest point method (see [Grenier 1988] and [Terras 1988, §4,4]). For example, let $\mathrm{k}=\mathbb{Q}$, $G=\mathrm{GL}_{n}$ and $Q$ be a standard maximal $\mathbb{Q}$-parabolic subgroup such that $Q \backslash G$ is a projective space. Then our construction gives a fundamental domain $\Omega_{Q}$ whose Archimedean part is isomorphic with Grenier's fundamental domain. If we choose another standard maximal $\mathbb{Q}$-parabolic subgroup of $\mathrm{GL}_{n}$ as $Q$, then the 
Archimedean part of $\Omega_{Q}$ yields a new kind of fundamental domain of $\mathrm{P}_{n}$ with respect to $\mathrm{GL}_{n}(\mathbb{Z})$ (see Example 3 in Section 7).

Notation. For a given ring $\mathfrak{A}$, the set of all $n$ by $k$ matrices with entries in $\mathfrak{A}$ is denoted by $\mathrm{M}_{n, k}(\mathfrak{A})$. We write $\mathrm{M}_{n}(\mathfrak{A})$ for $\mathrm{M}_{n, n}(\mathfrak{A})$. The transpose of a given matrix $a \in \mathrm{M}_{n, k}(\mathfrak{A})$ is denoted by ${ }^{t} a$. In this paper, $\mathrm{k}$ denotes an algebraic number field of finite degree over $\mathbb{Q}$ and o the ring of integers of $k$. The sets of all infinite and finite places of $\mathrm{k}$ are denoted by $\mathrm{p}_{\infty}$ and $\mathrm{p}_{f}$, respectively. For $\sigma \in \mathrm{p}_{\infty} \cup \mathrm{p}_{f}$, $\mathrm{k}_{\sigma}$ denotes the completion of $\mathrm{k}$ at $\sigma$. For $\sigma \in \mathrm{p}_{f}, \mathrm{o}_{\sigma}$ denotes the closure of $\mathrm{o}$ in $\mathrm{k}_{\sigma}$. The étale $\mathbb{R}$-algebra $\mathrm{k}_{\infty}=\mathrm{k} \otimes_{\mathbb{Q}} \mathbb{R}$ is identified with $\prod_{\sigma \in \mathrm{p}_{\infty}} \mathrm{k}_{\sigma}$. Let $\mathbb{A}$ and $\mathbb{A}^{x}$ denote the adele ring and the idèle group of $k$, respectively. The idèle norm of $\mathbb{A}^{x}$ is denoted by $|\cdot|$ A.

\section{Height functions}

Let $G$ be a connected affine algebraic group defined over k. For any k-algebra $\mathfrak{A}, G(\mathfrak{A})$ stands for the set of $\mathfrak{A}$-rational points of $G$. Let $X^{*}(G)_{\mathrm{k}}$ be the free $\mathbb{Z}$-module consisting of all k-rational characters of $G$. For each $g \in G(\mathbb{A})$, we define the homomorphism $\vartheta_{G}(g): X^{*}(G)_{\mathrm{k}} \rightarrow \mathbb{R}_{>0}$ by $\vartheta_{G}(g)(\chi)=|\chi(g)|_{\mathbb{A}}$ for $\chi \in X^{*}(G)_{\mathrm{k}}$. Then $\vartheta_{G}$ is a homomorphism from $G(\mathbb{A})$ into $\operatorname{Hom}_{\mathbb{Z}}\left(\boldsymbol{X}^{*}(G)_{\mathrm{k}}, \mathbb{R}_{>0}\right)$. We write $G(\mathbb{A})^{1}$ for the kernel of $\vartheta_{G}$.

In the following, let $G$ be a connected isotropic reductive group defined over k. We fix a maximal k-split torus $S$ of $G$ and a minimal k-parabolic subgroup $P_{0}$ of $G$ containing $S$. Denote by $\Phi_{\mathrm{k}}$ and $\Delta_{\mathrm{k}}$ the relative root system of $G$ with respect to $S$ and the set of simple roots of $\Phi_{\mathrm{k}}$ corresponding to $P_{0}$, respectively. Let $M_{0}$ be the centralizer of $S$ in $G$. Then $P_{0}$ has a Levi decomposition $P_{0}=M_{0} U_{0}$, where $U_{0}$ is the unipotent radical of $P_{0}$. A k-parabolic subgroup of $G$ containing $P_{0}$ is called a standard k-parabolic subgroup of $G$. Every standard k-parabolic subgroup $R$ of $G$ has a unique Levi subgroup $M_{R}$ containing $M_{0}$. We denote by $U_{R}$ the unipotent radical of $R$ and by $Z_{R}$ the greatest central $k$-split torus in $M_{R}$. Throughout this paper, we fix a maximal compact subgroup $K=\prod_{\sigma \in \mathrm{p}_{\infty}} K_{\sigma} \times \prod_{\sigma \in \mathrm{p}_{f}} K_{\sigma}$ of $G(\mathbb{A})$ satisfying the following property: for every standard k-parabolic subgroup $R$ of $G$, $K \cap M_{R}(\mathbb{A})$ is a maximal compact subgroup of $M_{R}(\mathbb{A})$, and $M_{R}(\mathbb{A})$ possesses an Iwasawa decomposition $\left(M_{R}(\mathbb{A}) \cap U_{0}(\mathbb{A})\right) M_{0}(\mathbb{A})\left(K \cap M_{R}(\mathbb{A})\right)$.

Let $Q$ be a standard proper maximal k-parabolic subgroup of $G$. There is only one simple root $\alpha_{0} \in \Delta_{\mathrm{k}}$ such that the restriction of $\alpha_{0}$ to $Z_{Q}$ is nontrivial. Let $n_{Q}$ be the positive integer such that $\left.n_{Q}^{-1} \alpha_{0}\right|_{Z_{Q}}$ is a $\mathbb{Z}$-basis of $X^{*}\left(Z_{Q} / Z_{G}\right)_{\mathrm{k}}$. We write

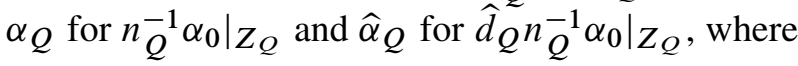

$$
\widehat{d}_{Q}=\left[\boldsymbol{X}^{*}\left(Z_{Q} / Z_{G}\right)_{\mathrm{k}}: \boldsymbol{X}^{*}\left(M_{Q} / Z_{G}\right)_{\mathrm{k}}\right] \text {. }
$$

Then $\hat{\alpha}_{Q}$ is a $\mathbb{Z}$-basis of the submodule $X^{*}\left(M_{Q} / Z_{G}\right)_{\mathrm{k}}$ of $X^{*}\left(Z_{Q} / Z_{G}\right)_{\mathrm{k}}$. Define 
the map $z_{Q}: G(\mathbb{A}) \rightarrow Z_{G}(\mathbb{A}) M_{Q}(\mathbb{A})^{1} \backslash M_{Q}(\mathbb{A})$ by $z_{Q}(g)=Z_{G}(\mathbb{A}) M_{Q}(\mathbb{A})^{1} m$ if $g=u m h$ with $u \in U_{Q}(\mathbb{A}), m \in M_{Q}(\mathbb{A})$ and $h \in K$. This is well defined and left $Z_{G}(\mathbb{A}) Q(\mathbb{A})^{1}$-invariant. Since $Z_{G}(\mathbb{A})^{1}=Z_{G}(\mathbb{A}) \cap G(\mathbb{A})^{1} \subset M_{Q}(\mathbb{A})^{1}, z_{Q}$ gives rise to a map from $Y_{Q}=Q(\mathbb{A})^{1} \backslash G(\mathbb{A})^{1}$ to $M_{Q}(\mathbb{A})^{1} \backslash\left(M_{Q}(\mathbb{A}) \cap G(\mathbb{A})^{1}\right)$. Namely, we have the following commutative diagram, whose vertical arrows are natural maps:

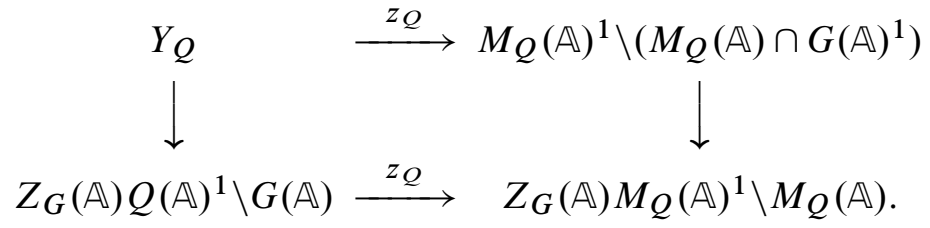

We define the height function $H_{Q}: G(\mathbb{A}) \rightarrow \mathbb{R}_{>0}$ by $H_{Q}(g)=\left|\hat{\alpha}_{Q}\left(z_{Q}(g)\right)\right|_{\mathbb{A}}^{-1}$ for $g \in G(\mathbb{A})$. We notice that the restriction of $H_{Q}$ to $M_{Q}(\mathbb{A})$ is a homomorphism from $M_{Q}(\mathbb{A})$ onto $\mathbb{R}_{>0}$.

Example 1. Let $G$ be a general linear group $\mathrm{GL}_{n}$ defined over the rational number field $\mathbb{Q}, P_{0}$ the group of upper triangular matrices in $G$ and $S$ the group of diagonal matrices in $G$. We fix an integer $k \in\{1, \ldots, n-1\}$, and let

$$
Q(\mathbb{Q})=\left\{\left(\begin{array}{ll}
a & b \\
0 & d
\end{array}\right): a \in \mathrm{GL}_{k}(\mathbb{Q}), b \in \mathrm{M}_{k, n-k}(\mathbb{Q}), d \in \mathrm{GL}_{n-k}(\mathbb{Q})\right\} .
$$

Then $Q$ is a standard maximal $\mathbb{Q}$-parabolic subgroup of $G$. The rational character $\widehat{\alpha}_{Q}$ and the height $H_{Q}$ are given by

$$
\widehat{\alpha}_{Q}\left(\left(\begin{array}{ll}
a & 0 \\
0 & d
\end{array}\right)\right)=(\operatorname{det} a)^{(n-k) / r}(\operatorname{det} d)^{-k / r}
$$

and

$$
H_{Q}\left(\left(\begin{array}{ll}
a & 0 \\
0 & d
\end{array}\right)\right)=|\operatorname{det} a|_{\mathbb{A}}^{-(n-k) / r}|\operatorname{det} d|_{\mathbb{A}}^{k / r},
$$

where $r$ denotes the greatest common divisor of $k$ and $n-k$. The height $H_{Q}$ has another expression. To explain this, let $\mathbb{Q}^{n}$ be an $n$-dimensional column vector space over $\mathbb{Q}$ with standard basis $\boldsymbol{e}_{1}, \ldots, \boldsymbol{e}_{n}$. The maximal parabolic subgroup $Q(\mathbb{Q})$ stabilizes the subspace spanned by $\boldsymbol{e}_{1}, \ldots, \boldsymbol{e}_{k}$. Let $V_{n, k}(\mathbb{Q})=\bigwedge^{k} \mathbb{Q}^{n}$ be the $k$-th exterior product of $\mathbb{Q}^{n}$. We set $V_{n, k}(\mathbb{A})=V_{n, k}(\mathbb{Q}) \otimes_{\mathbb{Q}} \mathbb{A}$ and $V_{n, k}\left(\mathbb{Q}_{\sigma}\right)=$ $V_{n, k}(\mathbb{Q}) \otimes_{\mathbb{Q}} \mathbb{Q}_{\sigma}$ for $\sigma \in \mathrm{p}_{\infty} \cup \mathrm{p}_{f}$. A $\mathbb{Q}$-basis of $V_{n, k}(\mathbb{Q})$ is formed by the elements $\boldsymbol{e}_{I}=\boldsymbol{e}_{i_{1}} \wedge \cdots \wedge \boldsymbol{e}_{i_{k}}$ with $I=\left\{i_{1}<i_{2}<\cdots<i_{k}\right\} \subset\{1, \ldots, n\}$. For a unique infinite place $\infty \in \mathrm{p}_{\infty}$, we define the local height $H_{\infty}: V_{n, k}\left(\mathbb{Q}_{\infty}\right) \rightarrow \mathbb{R}_{>0}$ by

$$
H_{\infty}\left(\sum_{I} a_{I} \boldsymbol{e}_{I}\right)=\left(\sum_{I}\left|a_{I}\right|_{\infty}^{2}\right)^{1 / 2},
$$


where $|\cdot|_{\infty}$ denotes the usual absolute value of $\mathbb{Q}_{\infty}=\mathbb{R}$. For each finite prime $p \in \mathrm{p}_{f}$, we define the local height $H_{p}: V_{n, k}\left(\mathbb{Q}_{p}\right) \rightarrow \mathbb{R}_{>0}$ by

$$
H_{p}\left(\sum_{I} a_{I} \boldsymbol{e}_{I}\right)=\sup _{I}\left|a_{I}\right|_{p},
$$

where $|\cdot|_{p}$ denotes the $p$-adic absolute value of $\mathbb{Q}_{p}$ normalized so that $|p|_{p}=p^{-1}$. Then the global height $H_{n, k}: V_{n, k}(\mathbb{Q}) \rightarrow \mathbb{R}_{>0}$ is defined to be a product of all local heights, that is, $H_{n, k}(x)=\prod_{\sigma \in \mathrm{p}_{\infty} \cup \mathrm{p}_{f}} H_{\sigma}(x)$ for $x \in V_{n, k}(\mathbb{Q})$. This $H_{n, k}$ is immediately extended to the subset $\operatorname{GL}\left(V_{n, k}(\mathbb{A})\right) V_{n, k}(\mathbb{Q})$ of the adele space $V_{n, k}(\mathbb{A})$ by

$$
H_{n, k}(A x)=\prod_{\sigma \in \mathrm{p}_{\infty} \cup \mathrm{p}_{f}} H_{\sigma}\left(A_{\sigma} x\right)
$$

for $A=\left(A_{\sigma}\right) \in \mathrm{GL}\left(V_{n, k}(\mathbb{A})\right)$ and $x \in V_{n, k}(\mathbb{Q})$. In particular, for $g \in G(\mathbb{A})=$ $\mathrm{GL}_{n}(\mathbb{A})$, we can take the value $H_{n, k}\left(g \boldsymbol{e}_{1} \wedge g \boldsymbol{e}_{2} \wedge \cdots \wedge g \boldsymbol{e}_{k}\right)$. We choose a maximal compact subgroup $K_{\infty}$ of $G\left(\mathbb{Q}_{\infty}\right)$ as $\left\{g \in G\left(\mathbb{Q}_{\infty}\right):{ }^{t} g^{-1}=g\right\}$. Let

$$
K_{f}=\prod_{p \in \mathrm{p}_{f}} \mathrm{GL}_{n}\left(\mathbb{Z}_{p}\right) \quad \text { and } \quad K=K_{\infty} \times K_{f}
$$

Then, by elementary computations, we have

$$
H_{n, k}\left(g \boldsymbol{e}_{1} \wedge g \boldsymbol{e}_{2} \wedge \cdots \wedge g \boldsymbol{e}_{k}\right)=|\operatorname{det} a|_{\mathbb{A}} \quad \text { if } g=h\left(\begin{array}{ll}
a & b \\
0 & d
\end{array}\right)
$$

with $h \in K, a \in \mathrm{GL}_{k}(\mathbb{A}), b \in \mathrm{M}_{k, n-k}(\mathbb{A})$ and $d \in \mathrm{GL}_{n-k}(\mathbb{A})$. Therefore, if $g \in G(\mathbb{A})^{1}$, that is, $|\operatorname{det} g|_{\mathbb{A}}=1$, then

$$
H_{Q}(g)=H_{n, k}\left(g^{-1} e_{1} \wedge g^{-1} e_{2} \wedge \cdots \wedge g^{-1} e_{k}\right)^{n / r} .
$$

\section{Twisted height functions restricted to one parameter subgroups}

Let $N_{G}(S)$ be the normalizer of $S$ in $G$ and $W_{G}=N_{G}(S)(\mathrm{k}) / M_{0}(\mathrm{k})$ the Weyl group of $G$ with respect to $S$. For a simple root $\alpha \in \Delta_{\mathrm{k}}, s_{\alpha} \in W_{G}$ denotes the simple reflection corresponding to $\alpha$. Then $\left\{s_{\alpha}\right\}_{\alpha \in \Delta_{\mathrm{k}}}$ generates $W_{G}$. We denote by $W_{G}^{Q}$ the subgroup of $W_{G}$ generated by $\left\{s_{\alpha}\right\}_{\alpha \in \Delta_{k} \backslash\left\{\alpha_{0}\right\}}$. For each $w \in W_{G}$, we use the same notation $w$ for a representative of $w$ in $N_{G}(S)(\mathrm{k})$. The following cell decomposition of $G(\mathrm{k})$ holds via Bruhat decomposition [Borel and Tits 1965, Proposition 4.10, Corollaire 5.20]:

$$
G(\mathrm{k})=\bigsqcup_{[w] \in W_{G}^{Q} \backslash W_{G} / W_{G}^{Q}} Q(\mathrm{k}) w Q(\mathrm{k}),
$$

where $[w]$ stands for the class $W_{G}^{Q} w W_{G}^{Q}$ in $W_{G}^{Q} \backslash W_{G} / W_{G}^{Q}$. 
The Weyl group $W_{G}$ acts on $\boldsymbol{X}^{*}(S)_{\mathrm{k}}$ by $w \cdot \chi: t \mapsto \chi\left(w^{-1} t w\right)$ for $w \in W_{G}$ and $\chi \in X^{*}(S)_{\mathrm{k}}$. We consider the restriction $\left.\hat{\alpha}_{Q}\right|_{S}$ of the rational character $\hat{\alpha}_{Q}$ of $M_{Q}$ to $S$.

Lemma 1. The subgroup of $W_{G}$ fixing $\left.\hat{\alpha}_{Q}\right|_{S}$ is equal to $W_{G}^{Q}$.

Proof. Put $W^{\prime}=\left\{w \in W_{G}:\left.w \cdot \hat{\alpha}_{Q}\right|_{S}=\left.\widehat{\alpha}_{Q}\right|_{S}\right\}$. Since a representative of $w \in W_{G}^{Q}$ is contained in $M_{Q}(\mathrm{k})$, we have $\widehat{\alpha}_{Q}\left(w^{-1} t w\right)=\widehat{\alpha}_{Q}(w)^{-1} \widehat{\alpha}_{Q}(t) \hat{\alpha}_{Q}(w)=\hat{\alpha}_{Q}(t)$ for all $t \in S$. Hence $W_{G}^{Q}$ is contained in $W^{\prime}$. By [Humphreys 1990, $\S 1.12$ Theorem (a) and (c)], $W^{\prime}$ is generated by a subset $W^{\prime} \cap\left\{s_{\alpha}\right\}_{\alpha \in \Delta_{\mathrm{k}}}$ of simple reflections. From $W_{G}^{Q} \subset W^{\prime}$, it follows $\left\{s_{\alpha}\right\}_{\alpha \in \Delta_{k} \backslash\left\{\alpha_{0}\right\}} \subset W^{\prime} \cap\left\{s_{\alpha}\right\}_{\alpha \in \Delta_{k}} \subset\left\{s_{\alpha}\right\}_{\alpha \in \Delta_{k}}$. Since $\widehat{\alpha}_{Q}$ is nontrivial on $S / Z_{G}, W^{\prime} \cap\left\{s_{\alpha}\right\}_{\alpha \in \Delta_{k}}$ must equal $\left\{s_{\alpha}\right\}_{\alpha \in \Delta_{k} \backslash\left\{\alpha_{0}\right\}}$. Therefore $W^{\prime}$ coincides with $W_{G}^{Q}$.

Let $\boldsymbol{X}_{*}(S)_{\mathrm{k}}$ be the free $\mathbb{Z}$-module consisting of all k-rational cocharacters of $S$. A natural pairing

$$
\langle\cdot, \cdot\rangle: X^{*}(S)_{\mathrm{k}} \times X_{*}(S)_{\mathrm{k}} \rightarrow \mathbb{Z}
$$

defined as in [Borel 1991, §8.6] is a regular pairing over $\mathbb{Z}$.

Lemma 2. Let $w_{1}$ and $w_{2}$ be elements of $W_{G}$ such that $w_{1}^{-1} W_{G}^{Q} \neq w_{2}^{-1} W_{G}^{Q}$. Then there exist a cocharacter $\xi=\xi_{w_{1}, w_{2}} \in X_{*}(S)_{\mathrm{k}}$ such that

$$
H_{Q}\left(w_{1} \xi(\lambda) w_{1}^{-1}\right)>H_{Q}\left(w_{2} \xi(\lambda) w_{2}^{-1}\right)
$$

holds for all $\lambda \in \mathbb{A}_{>1}^{\times}$, where $\mathbb{A}_{>1}^{\times}$denotes the set of $\lambda \in \mathbb{A}^{\times}$satisfying $|\lambda|_{\mathbb{A}}>1$.

Proof. Since $\left.w_{1}^{-1} \cdot \widehat{\alpha}_{Q}\right|_{S}-\left.w_{2}^{-1} \cdot \widehat{\alpha}_{Q}\right|_{S} \neq 0$ by Lemma 1 , there is a $\xi \in X_{*}(S)_{\mathrm{k}}$ such that $\left\langle\left. w_{1}^{-1} \cdot \hat{\alpha}_{Q}\right|_{S}-\left.w_{2}^{-1} \cdot \widehat{\alpha}_{Q}\right|_{S}, \xi\right\rangle<0$. The value $\ell=\left\langle\left. w_{1}^{-1} \cdot \widehat{\alpha}_{Q}\right|_{S}-\left.w_{2}^{-1} \cdot \widehat{\alpha}_{Q}\right|_{S}, \xi\right\rangle$ is a negative integer. We have

$$
\widehat{\alpha}_{Q}\left(w_{1} \xi(\lambda) w_{1}^{-1}\right) \cdot \hat{\alpha}_{Q}\left(w_{2} \xi(\lambda) w_{2}^{-1}\right)^{-1}=\lambda^{\ell}
$$

for all $\lambda \in \boldsymbol{G}_{m}$. Therefore,

$$
H_{Q}\left(w_{1} \xi(\lambda) w_{1}^{-1}\right) H_{Q}\left(w_{2} \xi(\lambda) w_{2}^{-1}\right)^{-1}=|\lambda|_{\AA}^{-\ell}>1
$$

holds for all $\lambda \in \mathbb{A}_{>1}^{\times}$.

\section{The Hermite function associated to $Q$ and minimal points}

We set $X_{Q}=Q(\mathrm{k}) \backslash G(\mathrm{k})$, which is regarded as a subset of $Y_{Q}=Q(\mathbb{A})^{1} \backslash G(\mathbb{A})^{1}$. Let $\pi_{X}: G(\mathrm{k}) \rightarrow X_{Q}$ be the natural quotient map. The symbol $\bar{e}=\pi_{X}(e) \in X_{Q}$ denotes the class of the unit element $e \in G(\mathrm{k})$. The Hermite function

$$
\mathrm{m}_{Q}: G(\mathbb{A})^{1} \rightarrow \mathbb{R}_{>0}
$$


is defined to be

$$
\mathrm{m}_{Q}(g)=\min _{x \in X_{Q}} H_{Q}(x g) .
$$

By definition, $\mathrm{m}_{Q}$ is a positive valued continuous function on $G(\mathrm{k}) \backslash G(\mathbb{A})^{1} / K$.

For each $g \in G(\mathbb{A})^{1}$, we put

$$
X_{Q}(g)=\left\{x \in X_{Q}: \mathrm{m}_{Q}(g)=H_{Q}(x g)\right\},
$$

which is a finite subset of $X_{Q}$. Thus we can define the counting function $\mathrm{n}_{Q}(g)=$ $\# X_{Q}(g)$.

Lemma 3. For any $g \in G(\mathbb{A})^{1}, \gamma \in G(\mathrm{k})$ and $h \in K$, one has $X_{Q}(\gamma g h)=$ $X_{Q}(g) \gamma^{-1}$. Especially, the counting function $\mathrm{n}_{Q}$ is left $G(\mathrm{k})$-invariant and right $K$-invariant.

The following lemma is proved by the same method as in [Watanabe 2012, Proof of Proposition 4.1].

Lemma 4. For $g \in G(\mathbb{A})^{1}$, there is a neighborhood $U$ of $g$ in $G(\mathbb{A})^{1}$ such that $X_{Q}\left(g^{\prime}\right) \subset X_{Q}(g)$ for all $g^{\prime} \in \mathcal{U}$.

Example 2. Let $G$ be a general linear group $\mathrm{GL}_{n}$ defined over $\mathbb{Q}$. We keep notations used in Example 1. In this case, we can express $m_{Q}$ in terms of some minimum of positive definite symmetric matrices. Since $\mathrm{GL}_{n} / \mathbb{Q}$ is of class number one, $G(\mathbb{A})^{1}=\left\{g \in \mathrm{GL}_{n}(\mathbb{A}):|\operatorname{det} g|_{\mathbb{A}}=1\right\}$ has the following decomposition:

$$
G(\mathbb{A})^{1}=G(\mathbb{Q})\left(G\left(\mathbb{Q}_{\infty}\right)^{1} \times K_{f}\right),
$$

where $G\left(\mathbb{Q}_{\infty}\right)^{1}=\left\{g \in \mathrm{GL}_{n}\left(\mathbb{Q}_{\infty}\right): \operatorname{det} g= \pm 1\right\}$ and $K_{f}=\prod_{p \in \mathrm{p}_{f}} \mathrm{GL}_{n}\left(\mathbb{Z}_{p}\right)$. We fix $g=\delta\left(g_{\infty} \times g_{f}\right) \in G(\mathbb{A})^{1}$ with $\delta \in G(\mathbb{Q}), g_{\infty} \in G\left(\mathbb{Q}_{\infty}\right)^{1}$ and $g_{f} \in K_{f}$. From the left $G(\mathbb{Q})$-invariance and the right $K$-invariance of $m_{Q}$, it follows that

$$
m_{Q}(g)=m_{Q}\left(g_{\infty}\right)=\min _{x \in X_{Q}} H_{Q}\left(x g_{\infty}\right)=\min _{\gamma \in G(\mathbb{Q})} H_{Q}\left(\gamma g_{\infty}\right) .
$$

Furthermore, since $G(\mathbb{Q})=Q(\mathbb{Q}) \mathrm{GL}_{n}(\mathbb{Z})$ and $H_{Q}$ is left $Q(\mathbb{Q})$-invariant, we have

$$
m_{Q}(g)=\min _{\gamma \in \mathrm{GL}_{n}(\mathbb{Z})} H_{Q}\left(\gamma g_{\infty}\right) .
$$

An elementary proof of the decomposition $G(\mathbb{Q})=Q(\mathbb{Q}) \mathrm{GL}_{n}(\mathbb{Z})$ is found in [Shimura 1994, Theorem 3]. By Example 1,

$$
\begin{aligned}
& H_{Q}\left(\gamma g_{\infty}\right)=H_{n, k}\left(g_{\infty}^{-1} \gamma^{-1} \boldsymbol{e}_{1} \wedge \cdots \wedge g_{\infty}^{-1} \gamma^{-1} \boldsymbol{e}_{k}\right)^{n / r} \\
& \quad=H_{\infty}\left(g_{\infty}^{-1} \gamma^{-1} \boldsymbol{e}_{1} \wedge \cdots \wedge g_{\infty}^{-1} \gamma^{-1} \boldsymbol{e}_{k}\right)^{n / r} \prod_{p \in \mathrm{p}_{f}} H_{p}\left(\gamma^{-1} \boldsymbol{e}_{1} \wedge \cdots \wedge \gamma^{-1} \boldsymbol{e}_{k}\right)^{n / r} \\
& \quad=H_{\infty}\left(g_{\infty}^{-1} \gamma^{-1} \boldsymbol{e}_{1} \wedge \cdots \wedge g_{\infty}^{-1} \gamma^{-1} \boldsymbol{e}_{k}\right)^{n / r} .
\end{aligned}
$$


Here we notice that $H_{p}\left(\gamma^{-1} \boldsymbol{e}_{1} \wedge \cdots \wedge \gamma^{-1} \boldsymbol{e}_{k}\right)=1$ for all $p \in \mathrm{p}_{f}$ and $\gamma \in \mathrm{GL}_{n}(\mathbb{Z})$. For a given $\gamma \in \mathrm{GL}_{n}(\mathbb{Z}), X_{\gamma}$ stands for the $n$ by $k$ matrix consisting of the first $k$ columns of $\gamma$. Binet's formula (see [Bombieri and Gubler 2006, Proposition 2.8.8]) yields

$$
H_{\infty}\left(g_{\infty}^{-1} \gamma^{-1} e_{1} \wedge \cdots \wedge g_{\infty}^{-1} \gamma^{-1} e_{k}\right)=\operatorname{det}\left({ }^{t} X_{\gamma^{-1}}{ }^{t} g_{\infty}^{-1} g_{\infty}^{-1} X_{\gamma^{-1}}\right)^{1 / 2} .
$$

As a consequence, we obtain

$$
m_{Q}(g)=\min _{X \in \mathrm{M}_{n, k}(\mathbb{Z})^{*}} \operatorname{det}\left({ }^{t} X^{t} g_{\infty}^{-1} g_{\infty}^{-1} X\right)^{n / 2 r},
$$

where $\mathrm{M}_{n, k}(\mathbb{Z})^{*}$ denotes the set of $X_{\gamma}$ for all $\gamma \in \mathrm{GL}_{n}(\mathbb{Z})$. In the case of $k=1$, $\mathrm{M}_{n, 1}(\mathbb{Z})^{*}$ is just the set of primitive vectors of the lattice $\mathbb{Z}^{n}$, and hence $m_{Q}(g)$ coincides with the $n / 2$ power of the arithmetical minimum of the positive definite symmetric matrix ${ }^{t} g_{\infty}^{-1} g_{\infty}^{-1}$.

\section{The Ryshkov domain of $G$ associated to $Q$}

We define the Ryshkov domain $\mathrm{R}=\mathrm{R}\left(\mathrm{m}_{Q}\right)$ of $\mathrm{m}_{Q}$ by

$$
\mathrm{R}=\mathrm{R}\left(\mathrm{m}_{Q}\right)=\left\{g \in G(\mathbb{A})^{1}: \mathrm{m}_{Q}(g) / H_{Q}(g) \geq 1\right\} .
$$

Since $\mathrm{m}_{Q}(g) \leq H_{Q}(g)$ holds for all $g \in G(\mathbb{A})^{1}$, we have

$$
\begin{aligned}
\mathrm{R} & =\left\{g \in G(\mathbb{A})^{1}: \mathrm{m}_{Q}(g)=H_{Q}(g)\right\} \\
& =\left\{g \in G(\mathbb{A})^{1}: \bar{e} \in X_{Q}(g)\right\} .
\end{aligned}
$$

Since both $H_{Q}$ and $\mathrm{m}_{Q}$ are continuous, $\mathrm{R}$ is a closed subset in $G(\mathbb{A})^{1}$.

Lemma 5. One has $Q(\mathrm{k}) \mathrm{R} K=\mathrm{R}$ and $G(\mathbb{A})^{1}=G(\mathrm{k}) \mathrm{R}$.

Proof. The first assertion is obvious by the definition of $H_{Q}$. To prove the second assertion, we choose a minimal point $x \in X_{Q}(g)$ for a given $g \in G(\mathbb{A})^{1}$. There is a $\gamma \in G(\mathrm{k})$ such that $x=\pi_{X}(\gamma)$. Then $H_{Q}(x g)=H_{Q}(\gamma g)=\mathrm{m}_{Q}(g)=\mathrm{m}_{Q}(\gamma g)$ since $\mathrm{m}_{Q}$ is left $G(\mathrm{k})$-invariant. Therefore, $\gamma g \in \mathrm{R}$.

Lemma 6. Let $C$ be an arbitrary subset of $G(\mathbb{A})^{1}$, and let $g \in G(\mathbb{A})^{1}$ and $\gamma \in G(\mathrm{k})$.

(1) $\gamma g \in \mathrm{R}$ if and only if $\pi_{X}(\gamma) \in X_{Q}(g)$.

(2) $X_{Q}(g)=\pi_{X}(\{\gamma \in G(\mathrm{k}): \gamma g \in \mathrm{R}\})$.

(3) $\gamma C \subset \mathrm{R}$ if and only if $\pi_{X}(\gamma) \in \bigcap_{g \in C} X_{Q}(g)$.

(4) $\bigcap_{g \in \mathrm{R}} X_{Q}(g)=\{\bar{e}\}$.

(5) $\gamma \mathrm{R} \subset \mathrm{R}$ if and only if $\gamma \in Q(\mathrm{k})$. 
Proof. By definition, $\gamma g \in \mathrm{R}$ if and only if $\mathrm{m}_{Q}(\gamma g)=H_{Q}(\gamma g)$. This is equivalent to $\pi_{X}(\gamma) \in X_{Q}(g)$ because $\mathrm{m}_{Q}(\gamma g)=\mathrm{m}_{Q}(g)$. Both (2) and (3) follow from (1). For a point $x=\pi_{X}(\gamma) \in \bigcap_{g \in \mathrm{R}} X_{Q}(g)$, we have $\gamma Q(\mathrm{k}) \mathrm{R} \subset \mathrm{R}$; in other words, $x Q(\mathrm{k}) \subset$ $\bigcap_{g \in \mathrm{R}} X_{Q}(g)$. Since $x Q(\mathrm{k})$ is an infinite set for $x \neq \bar{e}$ by Bruhat decomposition, we must have $x=\bar{e}$. This shows (4). Item (5) follows from (3) and (4).

Lemma 7. Let $g_{0} \in \mathrm{R}$ be an element such that $\mathrm{n}_{Q}\left(g_{0}\right)>1$ and $x_{0}$ an arbitrary element in $X_{Q}\left(g_{0}\right)$. Then, any neighborhood $U$ of $g_{0}$ in $G(\mathbb{A})^{1}$ contains a point $g$ such that $X_{Q}(g) \subset X_{Q}\left(g_{0}\right)$ and $x_{0} \notin X_{Q}(g)$.

Proof. We may assume $U$ satisfies $X_{Q}(g) \subset X_{Q}\left(g_{0}\right)$ for all $g \in \mathcal{U}$ by Lemma 4. Since $\mathrm{n}_{Q}\left(g_{0}\right)>1$, there is an $x \in X_{Q}\left(g_{0}\right)$ such that $x \neq \bar{e}$. This $x$ is of the form $\pi_{X}(w \gamma)$ with $w \in W_{G} \backslash W_{G}^{Q}$ and $\gamma \in Q(\mathrm{k})$. By Lemma 2, there is a cocharacter $\xi=\xi_{w, e} \in X_{*}(S)_{\mathrm{k}}$ such that $H_{Q}\left(w \xi(\lambda) w^{-1}\right)>H_{Q}(\xi(\lambda))$ holds for all $\lambda \in \mathbb{A}_{>1}^{\times}$. Let $\lambda \in \mathbb{A}^{\times}$be an element sufficiently close to 1 so that $g_{\lambda}=\gamma^{-1} \xi(\lambda) \gamma g_{0}$ is contained in $U$. We have

$$
\begin{aligned}
H_{Q}\left(g_{\lambda}\right) & =H_{Q}\left(\xi(\lambda) \gamma g_{0}\right)=H_{Q}(\xi(\lambda)) H_{Q}\left(\gamma g_{0}\right) \\
& =H_{Q}(\xi(\lambda)) H_{Q}\left(g_{0}\right)=H_{Q}(\xi(\lambda)) \mathrm{m}_{Q}\left(g_{0}\right)
\end{aligned}
$$

and

$$
\begin{aligned}
H_{Q}\left(x g_{\lambda}\right) & =H_{Q}\left(w \xi(\lambda) \gamma g_{0}\right)=H_{Q}\left(w \xi(\lambda) w^{-1}\right) H_{Q}\left(w \gamma g_{0}\right) \\
& =H_{Q}\left(w \xi(\lambda) w^{-1}\right) \mathrm{m}_{Q}\left(g_{0}\right) .
\end{aligned}
$$

If $x_{0}=\bar{e}$, then we choose $\lambda$ sufficiently close to 1 satisfying $\lambda^{-1} \in \mathbb{A}_{>1}^{\times}$. Since $X_{Q}\left(g_{\lambda}\right) \subset X_{Q}\left(g_{0}\right)$ and $\mathrm{m}_{Q}\left(g_{\lambda}\right) \leq H_{Q}\left(x g_{\lambda}\right)<H_{Q}\left(g_{\lambda}\right), X_{Q}\left(g_{\lambda}\right)$ does not contain $\bar{e}$. If $x_{0} \neq \bar{e}$, then we choose $x$ as $x_{0}$ and $\lambda \in \mathbb{A}_{>1}^{\times}$sufficiently close to 1 . Since $\mathrm{m}_{Q}\left(g_{\lambda}\right) \leq H_{Q}\left(g_{\lambda}\right)<H_{Q}\left(x_{0} g_{\lambda}\right), X_{Q}\left(g_{\lambda}\right)$ does not contain $x_{0}$.

Lemma 8. $\min _{g \in G(\mathbb{A})}{ }^{1} \mathrm{n}_{Q}(g)=\min _{g \in \mathrm{R}} \mathrm{n}_{Q}(g)=1$.

Proof. From Lemma 5 and the $G(\mathrm{k})$-invariance of $\mathrm{n}_{Q}$, it follows that

$$
\min _{g \in G(\mathbb{A})^{1}} \mathrm{n}_{Q}(g)=\min _{g \in \mathrm{R}} \mathrm{n}_{Q}(g) .
$$

If $g_{0} \in$ R satisfies $\min _{g \in \mathrm{R}} \mathrm{n}_{Q}(g)=\mathrm{n}_{Q}\left(g_{0}\right)>1$, then by Lemmas 5 and 7 , there exist a point $g_{1} \in G(\mathbb{A})^{1}$ and $\gamma_{1} \in G(\mathrm{k})$ such that $\mathrm{n}_{Q}\left(\gamma_{1} g_{1}\right)=\mathrm{n}_{Q}\left(g_{1}\right)<\mathrm{n}_{Q}\left(g_{0}\right)$ and $\gamma_{1} g_{1} \in \mathrm{R}$. This is a contradiction.

We define the subset $R_{1}$ of $R$ by

$$
\mathrm{R}_{1}=\left\{g \in \mathrm{R}: \mathrm{n}_{Q}(g)=1\right\}=\left\{g \in G(\mathbb{A})^{1}: X_{Q}(g)=\{\bar{e}\}\right\} .
$$

Lemma 9. $\mathrm{R}_{1}$ coincides with the interior $\mathrm{R}^{\circ}$ of $\mathrm{R}$ in $G(\mathbb{A})^{1}$. 
Proof. For $g \in \mathrm{R}_{1}$, we choose a neighborhood $U$ of $g$ in $G(\mathbb{A})^{1}$ as in Lemma 4. Then $u \subset R_{1}$. Therefore, $R_{1}$ is open and is contained in $R^{\circ}$. If there exists an element $g_{0} \in \mathrm{R}^{\circ}$ such that $\mathrm{n}_{Q}\left(g_{0}\right)>1$, then, by Lemma $7, \mathrm{R}^{\circ}$ contains an element $g$ satisfying $\bar{e} \notin X_{Q}(g)$. This contradicts $g \in \mathrm{R}$.

It is obvious that $G(\mathrm{k}) \mathrm{R}_{1}=\left\{g \in G(\mathbb{A})^{1}: \mathrm{n}_{Q}(g)=1\right\}$.

Lemma 10. $G(\mathrm{k}) \mathrm{R}_{1}$ is open and dense in $G(\mathbb{A})^{1}$.

Proof. Since $\mathrm{R}_{1}$ is open in $G(\mathbb{A})^{1}$, so is $G(\mathrm{k}) \mathrm{R}_{1}$. We assume $G(\mathbb{A})^{1} \backslash G(\mathrm{k}) \mathrm{R}_{1}$ has an interior point $g_{0}$. Let $U$ be a neighborhood of $g_{0}$ in $G(\mathbb{A})^{1}$ so that $U \cap G(\mathrm{k}) \mathrm{R}_{1}=\varnothing$. By Lemma 5, we can take $\gamma_{0} \in G(\mathrm{k})$ such that $\gamma_{0} g_{0} \in \mathrm{R}$. Since $\mathrm{n}_{Q}\left(\gamma_{0} g_{0}\right)=$ $\mathrm{n}_{Q}\left(g_{0}\right)>1$, by Lemmas 5 and 7, there exist $g_{1} \in \gamma_{0} u$ and $\gamma_{1} \in G(\mathrm{k})$ such that $\mathrm{n}_{Q}\left(g_{1}\right)<\mathrm{n}_{Q}\left(g_{0}\right)$ and $\gamma_{1} g_{1} \in \mathrm{R}$. If $\mathrm{n}_{Q}\left(g_{1}\right)>1$, then there exist $g_{2} \in \gamma_{1} \gamma_{0} u$ and $\gamma_{2} \in G(\mathrm{k})$ such that $\mathrm{n}_{Q}\left(g_{2}\right)<\mathrm{n}_{Q}\left(g_{1}\right)$ and $\gamma_{2} g_{2} \in R$. This process terminates after finitely many iterations. At the last step, we obtain an element $g_{\ell} \in \gamma_{\ell-1} \cdots \gamma_{0} u$ such that $\mathrm{n}_{Q}\left(g_{\ell}\right)=1$. Then $\left(\gamma_{\ell-1} \cdots \gamma_{0}\right)^{-1} g_{\ell}$ is contained in $U \cap G(\mathrm{k}) \mathrm{R}_{1}$. This contradicts $U \cap G(\mathrm{k}) \mathrm{R}_{1}=\varnothing$. Therefore, $G(\mathbb{A})^{1} \backslash G(\mathrm{k}) \mathrm{R}_{1}$ is nowhere dense in $G(\mathbb{A})^{1}$.

Lemma 11. For $\gamma \in G(\mathrm{k}), \mathrm{R}_{1} \cap \gamma \mathrm{R} \neq \varnothing$ if and only if $\gamma \in Q(\mathrm{k})$.

Proof. If $\mathrm{R}_{1} \cap \gamma \mathrm{R}$ has an element $g$, then $\pi_{X}\left(\gamma^{-1}\right) \in X_{Q}(g)=\{\bar{e}\}$ by Lemma 6 .

Lemma 12. Let $\mathrm{R}_{1}^{-}$be the closure of $\mathrm{R}_{1}$. Then we have the following subdivision of $G(\mathbb{A})^{1}$ :

$$
G(\mathbb{A})^{1}=\bigcup_{\gamma Q(\mathrm{k}) \in G(\mathrm{k}) / Q(\mathrm{k})} \gamma \mathrm{R}_{1}^{-}
$$

Proof. We fix an arbitrary $g \in G(\mathbb{A})^{1}$. By Lemma 10 , there exists a sequence $\left\{g_{n}\right\} \subset G(\mathrm{k}) \mathrm{R}_{1}$ such that $\lim _{n \rightarrow \infty} g_{n}=g$. We take a neighborhood $\mathcal{U}$ of $g$ as in Lemma 4 and may assume that $\left\{g_{n}\right\} \subset \mathcal{U}$. Since $g_{n} \in G(\mathrm{k}) \mathrm{R}_{1}, X_{Q}\left(g_{n}\right)$ consists of a single element $\pi_{X}\left(\gamma_{n}\right)$, where $\gamma_{n} \in G(\mathrm{k})$. From $g_{n} \in \mathcal{U}$, it follows that $\pi_{X}\left(\gamma_{n}\right) \in X_{Q}(g)$ for all $n$. Since $X_{Q}(g)$ is a finite set, we can take a subsequence $\left\{g_{n_{j}}\right\}$ such that $\pi_{X}\left(\gamma_{n_{j}}\right)=\pi_{X}(\gamma) \in X_{Q}(g)$ for all $n_{j}$. Then $\left\{g_{n_{j}}\right\} \subset \gamma^{-1} \mathrm{R}_{1}$, and $g$ is contained in the closure of $\gamma^{-1} \mathrm{R}_{1}$.

For $g \in G(\mathbb{A})^{1}$, we put

$$
S_{Q}(g)=\pi_{X}\left(\left\{\gamma \in G(\mathrm{k}): \gamma g \in \mathrm{R}_{1}^{-}\right\}\right) .
$$

By Lemmas 6 and 12, $S_{Q}(g)$ is a nonempty subset of $X_{Q}(g)$.

Lemma 13. For $g_{0} \in G(\mathbb{A})^{1}$, there is a neighborhood $U$ of $g_{0}$ in $G(\mathbb{A})^{1}$ such that $S_{Q}(g) \subset S_{Q}\left(g_{0}\right)$ for all $g \in \mathcal{U}$. 
Proof. Let $U$ be a neighborhood of $g_{0}$ such that $X_{Q}(g) \subset X_{Q}\left(g_{0}\right)$ for all $g \in \mathcal{U}$. Since $g_{0} \notin \gamma^{-1} \mathrm{R}_{1}^{-}$for any $\pi_{X}(\gamma) \in X_{Q}\left(g_{0}\right) \backslash S_{Q}\left(g_{0}\right)$, we can take a sufficiently small $u$ so that $U \cap \gamma^{-1} \mathrm{R}_{1}^{-}=\varnothing$ for all $\pi_{X}(\gamma) \in X_{Q}\left(g_{0}\right) \backslash S_{Q}\left(g_{0}\right)$. Then, for any $g \in \mathcal{U}, S_{Q}(g) \cap X_{Q}\left(g_{0}\right) \backslash S_{Q}\left(g_{0}\right)$ is empty; that is, $S_{Q}(g) \subset S_{Q}\left(g_{0}\right)$.

Remark. We do not know whether $\mathrm{R}_{1}^{-}=\mathrm{R}$ holds or not in general. If it does, then $S_{Q}(g)=X_{Q}(g)$ holds for all $g$.

\section{A fundamental domain of $G(\mathbb{A})^{1}$ with respect to $G(\mathrm{k})$}

Definition. Let $T$ be a locally compact Hausdorff space and $\Gamma$ be a discrete group acting on $T$ from the left. Assume that the action of $\Gamma$ on $T$ is properly discontinuous. An open subset $\Omega$ of $T$ is called an open fundamental domain of $T$ with respect to $\Gamma$ if $\Omega$ satisfies the following conditions:

(1) $T=\Gamma \Omega^{-}$, where $\Omega^{-}$stands for the closure of $\Omega$ in $T$, and

(2) $\Omega \cap \gamma \Omega^{-}=\varnothing$ if $\gamma \in \Gamma \backslash\{e\}$.

A subset $\mathrm{F}$ of $T$ is called a fundamental domain of $T$ with respect to $\Gamma$ if there is an open fundamental domain $\Omega$ as above such that $\Omega \subset \mathrm{F} \subset \Omega^{-}$.

By Baer and Levi's theorem [1931] (see also [van der Waerden 1935, §10]), an open fundamental domain of $T$ with respect to $\Gamma$ exists if the set of points stabilized by some nontrivial element of $\Gamma$ is discrete in $T$. Thus there exists an open fundamental domain $\Omega_{Q}$ of $\mathrm{R}_{1}^{-}$with respect to $Q(\mathrm{k})$. For a given subset $A$ of $\mathrm{R}_{1}^{-}, A^{\circ}$ and $A^{-}$denote the interior and the closure of $A$ in $G(\mathbb{A})^{1}$, respectively. Since $\mathrm{R}_{1}^{-}$is closed in $G(\mathbb{A})^{1}$, the closure of $A$ in $\mathrm{R}_{1}^{-}$coincides with $A^{-}$.

Lemma 14. Let $\Omega_{Q}$ be an open fundamental domain of $\mathrm{R}_{1}^{-}$with respect to $Q(\mathrm{k})$. Then one has $\Omega_{Q}^{\circ}=\Omega_{Q} \cap \mathrm{R}_{1}$ and $\Omega_{Q}^{-}=\left(\Omega_{Q} \cap \mathrm{R}_{1}\right)^{-}$.

Proof. Since $\Omega_{Q}$ is an open set in $\mathrm{R}_{1}^{-}$with respect to the relative topology, there is an open set $U$ in $G(\mathbb{A})^{1}$ such that $\Omega_{Q}=\mathrm{R}_{1}^{-} \cap U$. Therefore, $\Omega_{Q} \cap \mathrm{R}_{1}=U \cap \mathrm{R}_{1}$ is open in $G(\mathbb{A})^{1}$, and hence $\Omega_{Q}^{\circ}=\Omega_{Q} \cap \mathrm{R}_{1}$. Since $\mathrm{R}_{1}$ is dense in $\mathrm{R}_{1}^{-}$and $\Omega_{Q}$ is relatively open in $\mathrm{R}_{1}^{-}$, the closure of $\Omega_{Q} \cap \mathrm{R}_{1}$ in $\mathrm{R}_{1}^{-}$contains $\Omega_{Q}$, that is, $\Omega_{Q} \subset\left(\Omega_{Q} \cap \mathrm{R}_{1}\right)^{-}$. Hence $\Omega_{Q}^{-}=\left(\Omega_{Q} \cap \mathrm{R}_{1}\right)^{-}$.

Theorem 15. Let $\Omega_{Q}$ be an open fundamental domain of $\mathrm{R}_{1}^{-}$with respect to $Q(\mathrm{k})$. Then $\Omega_{Q}^{\circ}$ is an open fundamental domain of $G(\mathbb{A})^{1}$ with respect to $G(\mathrm{k})$.

Proof. From $\mathrm{R}_{1}^{-}=Q(\mathrm{k}) \Omega_{Q}^{-}$and Lemma 12, it follows $G(\mathbb{A})^{1}=G(\mathrm{k}) \Omega_{Q}^{-}$. For $\gamma \in G(\mathrm{k})$, we assume $\Omega_{Q}^{\circ} \cap \gamma \Omega_{Q}^{-} \neq \varnothing$. By Lemma 11, $\gamma$ is contained in $Q(\mathrm{k})$. Since $\Omega_{Q}$ is an open fundamental domain of $\mathrm{R}_{1}^{-}$with respect to $Q(\mathrm{k}), \gamma$ must be equal to $e$.

For a given subset $A$ of $G(\mathbb{A})^{1}$, we denote by $\partial A$ the boundary of $A$. 
Lemma 16. If $g_{0} \in \mathrm{R}_{1}^{-}$attains a local maximum of $\mathrm{m}_{Q}$, then $g_{0}$ is in $\partial \mathrm{R}_{1}^{-}$.

Proof. Suppose $g_{0} \in \mathrm{R}_{1}$. Since $\mathrm{R}_{1}$ is open, $z g_{0}$ is contained in $\mathrm{R}_{1}$ if $z \in Z_{Q}(\mathbb{A})$ is sufficiently close to $e$. Then

$$
\mathrm{m}_{Q}\left(z g_{0}\right)=H_{Q}\left(z g_{0}\right)=H_{Q}(z) H_{Q}\left(g_{0}\right)=H_{Q}(z) \mathrm{m}_{Q}\left(g_{0}\right) .
$$

Since $H_{Q}(z)$ can vary on the interval $(1-\epsilon, 1+\epsilon)$ for a sufficiently small $\epsilon>0$, $\mathrm{m}_{Q}\left(g_{0}\right)$ is not a local maximum of $\mathrm{m}_{Q}$.

Since $\left(\Omega_{Q}^{-}\right)^{\circ}=\Omega_{Q}^{\circ} \subset \mathrm{R}_{1}$, the following theorem immediately follows from Lemma 16.

Theorem 17. Let $\Omega_{Q}$ be the same as in Theorem 15. If $g_{0} \in \Omega_{Q}^{-}$attains a local maximum of $\mathrm{m}_{Q}$, then $g_{0}$ is in $\partial \Omega_{Q}^{-} \cap \partial \mathrm{R}_{1}^{-}$.

Remark. A point $g_{0} \in G(\mathbb{A})^{1}$ is said to be extreme if $g_{0}$ attains a local maximum of $\mathrm{m}_{Q}$. By Theorem 17, any extreme point is contained in $G(\mathrm{k})\left(\partial \Omega_{Q}^{-} \cap \partial \mathrm{R}_{1}^{-}\right)$. A candidate of the notion analogous to perfect quadratic forms is the following: a point $g \in G(\mathbb{A})^{1}$ is said to be $Q$-perfect if there is a neighborhood $U$ of $g$ such that

$$
u \cap \bigcap_{\pi_{X}(\delta) \in S_{Q}(g)} \delta^{-1} \mathrm{R}_{1}^{-}=\{g\} .
$$

\section{The case when $G$ is of class number one}

We put $K_{f}=\prod_{\sigma \in \mathrm{p}_{f}} K_{\sigma}, G_{\mathbb{A}, \infty}=G\left(\mathrm{k}_{\infty}\right) \times K_{f}, G_{\AA, \infty}^{1}=G_{\mathbb{A}, \infty} \cap G(\mathbb{A})^{1}$ and $G_{\mathrm{o}}=G(\mathrm{k}) \cap G_{\mathrm{A}, \infty}$. By identifying $G\left(\mathrm{k}_{\infty}\right)$ with the subgroup

$$
\left\{\left(g_{\sigma}\right) \in G(\mathbb{A}): g_{\sigma}=e \text { for all } \sigma \in \mathrm{p}_{f}\right\}
$$

of $G(\mathbb{A})$, we put $G\left(\mathrm{k}_{\infty}\right)^{1}=G\left(\mathrm{k}_{\infty}\right) \cap G(\mathbb{A})^{1}$. The number $n_{\mathrm{k}}(G)$ of double cosets in $G(\mathbb{A})$ modulo $G(\mathrm{k})$ and $G_{\mathbb{A}, \infty}$ is called the class number of $G$. For example, $n_{\mathrm{k}}\left(\mathrm{GL}_{n}\right)$ is equal to the class number of $\mathrm{k}$. If $G$ is almost k-simple, k-isotropic and simply connected, then $n_{\mathrm{k}}(G)=1$ by the strong approximation theorem. In this section, we assume that $n_{\mathrm{k}}(G)=1$. Then $G(\mathbb{A})^{1}=G(\mathrm{k}) G_{\mathrm{A}, \infty}^{1}$. Let $h_{Q}$ be the number of double cosets of $G(\mathrm{k})$ modulo $Q(\mathrm{k})$ and $G_{\mathrm{o}}$. By [Borel 1963, Proposition 7.5], $h_{Q}$ is equal to the class number of $M_{Q}$. Let $\left\{\xi_{1}=e, \xi_{2}, \ldots, \xi_{h_{Q}}\right\}$ be a complete system of representatives of $Q(\mathrm{k}) \backslash G(\mathrm{k}) / G_{\mathrm{o}}$. For each $\xi_{i}$, we define

$$
\mathrm{R}_{\xi_{i}, \infty}=\left\{g_{\infty} \in G\left(\mathrm{k}_{\infty}\right)^{1}: \mathrm{m}_{Q}\left(g_{\infty}\right)=H_{Q}\left(\xi_{i} g_{\infty}\right)\right\} .
$$

Since $G(\mathrm{k})$ is a disjoint union of $Q(\mathrm{k}) \xi_{i} G_{\mathrm{o}}$ for $i=1, \ldots, h_{Q}, \mathrm{~m}_{Q}\left(g_{\infty}\right)$ equals

$$
\min _{1 \leq i \leq h_{Q}} \min _{\delta \in G_{\circ}} H_{Q}\left(\xi_{i} \delta g_{\infty}\right) .
$$


Lemma 18.

$$
\mathrm{R}=\bigsqcup_{i=1}^{h_{Q}} Q(\mathrm{k}) \xi_{i}\left(\mathrm{R}_{\xi_{i}, \infty} \times K_{f}\right)
$$

Proof. For each $i, Q(\mathrm{k}) \xi_{i}\left(\mathrm{R}_{\xi_{i}, \infty} \times K_{f}\right) \subset \mathrm{R}$ is trivial. Since

$$
G(\mathbb{A})^{1}=\bigsqcup_{i=1}^{h_{Q}} Q(\mathrm{k}) \xi_{i} G_{\AA}^{1}, \infty
$$

by [Borel 1963, §7], a given $g \in \mathrm{R}$ is represented as $g=\gamma \xi_{i}\left(g_{\infty} \times g_{f}\right)$ for some $i, \gamma \in Q(\mathrm{k})$ and $g_{\infty} \times g_{f} \in G_{\mathrm{A}, \infty}^{1}$. Then $\mathrm{m}_{Q}(g)=H_{Q}(g)$ implies $\mathrm{m}_{Q}\left(g_{\infty}\right)=$ $H_{Q}\left(\xi_{i} g_{\infty}\right)$. Therefore, $g_{\infty} \in \mathrm{R}_{\xi_{i}, \infty}$.

We write $Q_{i}$ for the conjugate $\xi_{i}^{-1} Q \xi_{i}$ of $Q$. This $Q_{i}$ is a maximal k-parabolic subgroup of $G$. We put $Q_{i, \mathrm{o}}=Q_{i}(\mathrm{k}) \cap G_{\mathbb{A}, \infty}$.

Lemma 19. If $g\left(\mathrm{R}_{\xi_{i}, \infty} \times K_{f}\right) \cap\left(\mathrm{R}_{\xi_{i}, \infty} \times K_{f}\right)$ is nonempty for $g \in Q_{i}(\mathrm{k})$, then $g \in Q_{i, 0}$.

Proof. If there is an $h \in \mathrm{R}_{\xi_{i}, \infty} \times K_{f}$ such that $g h \in \mathrm{R}_{\xi_{i}, \infty} \times K_{f}$, then

$$
g \in\left(\mathrm{R}_{\xi_{i}, \infty} \times K_{f}\right) h^{-1} \subset G_{\AA, \infty} .
$$

It is easy to prove that the group $Q_{i, \mathrm{o}}$ stabilizes $\mathrm{R}_{\xi_{i}, \infty} \times K_{f}$ by left multiplication. We fix a complete system $\left\{\gamma_{i j}\right\}_{j}$ of representatives of $Q_{i}(\mathrm{k}) / Q_{i, \mathrm{o}}$. It follows from Lemma 19 that $\gamma_{i j}\left(\mathrm{R}_{\xi_{i}, \infty} \times K_{f}\right) \cap \gamma_{i k}\left(\mathrm{R}_{\xi_{i}, \infty} \times K_{f}\right)=\varnothing$ if $j \neq k$. Therefore, we obtain the following subdivision of $\mathrm{R}$ :

$$
\mathrm{R}=\bigsqcup_{i=1}^{h_{Q}} \bigsqcup_{j} \xi_{i} \gamma_{i j}\left(\mathrm{R}_{\xi_{i}, \infty} \times K_{f}\right) .
$$

Let $\mathrm{R}_{\xi_{i}, \infty}^{\circ}$ be the interior of $\mathrm{R}_{\xi_{i}, \infty}$ and $\mathrm{R}_{\xi_{i}, \infty}^{*}$ the closure of $\mathrm{R}_{\xi_{i}, \infty}^{\circ}$ in $G\left(\mathrm{k}_{\infty}\right)^{1}$. Since the union of (1) is disjoint, it is obvious that

$$
\mathrm{R}_{1}^{-}=\bigsqcup_{i=1}^{h_{Q}} \bigsqcup_{j} \xi_{i} \gamma_{i j}\left(\mathrm{R}_{\xi_{i}, \infty}^{*} \times K_{f}\right) .
$$

Proposition 20. Let $\Omega_{i, \infty}$ be an open fundamental domain of $\mathrm{R}_{\xi_{i}, \infty}^{*}$ with respect to $Q_{i, \mathrm{o}}$ for $i=1, \ldots, h_{Q}$. Then the set

$$
\Omega=\bigsqcup_{i=1}^{h_{Q}} \xi_{i}\left(\Omega_{i, \infty} \times K_{f}\right)
$$

gives an open fundamental domain of $\mathrm{R}_{1}^{-}$with respect to $Q(\mathrm{k})$. 
Proof. Let $\Omega_{i, \infty}^{-}$denote the closure of $\Omega_{i, \infty}$ in $G\left(\mathrm{k}_{\infty}\right)^{1}$. For $g \in Q(\mathrm{k})$, we assume $\Omega \cap g \Omega^{-} \neq \varnothing$. Then, for some $i, j$,

$$
\xi_{i}\left(\Omega_{i, \infty} \times K_{f}\right) \cap g \xi_{j}\left(\Omega_{j, \infty}^{-} \times K_{f}\right) \neq \varnothing .
$$

There exist $\gamma_{j k}$ and $\delta \in Q_{j, \circ}$ such that $\xi_{j}^{-1} g \xi_{j}=\gamma_{j k} \delta$. Then (3) is the same as

$$
\xi_{i}\left(\Omega_{i, \infty} \times K_{f}\right) \cap \xi_{j} \gamma_{j k}\left(\delta \Omega_{j, \infty}^{-} \times K_{f}\right) \neq \varnothing .
$$

By (1), we have $i=j, \gamma_{j k}=e$ and $\Omega_{j, \infty} \cap \delta \Omega_{j, \infty}^{-} \neq \varnothing$. Since $\Omega_{j, \infty}$ is an open fundamental domain of $\mathrm{R}_{\xi_{j}, \infty}^{*}$ with respect to $Q_{j, \mathrm{o}}, \delta$ must be equal to $e$. Therefore, $\Omega \cap g \Omega^{-} \neq \varnothing$ implies $g=e$. Finally, $Q(\mathrm{k}) \Omega^{-}=\mathrm{R}_{1}^{-}$follows from (2) and $Q_{i, \mathrm{o}} \Omega_{i, \infty}^{-}=\mathrm{R}_{\xi_{i}, \infty}^{*}$.

By Theorem 17, we obtain the following.

Corollary 21. If $g_{0} \in \Omega^{-}$attains a local maximum of $\mathrm{m}_{Q}$, then $g_{0}$ is contained in the set

$$
\bigsqcup_{i=1}^{h_{Q}} \xi_{i}\left(\left(\partial \Omega_{i, \infty}^{-} \cap \partial \mathrm{R}_{\xi_{i}, \infty}^{*}\right) \times K_{f}\right) .
$$

We consider the infinite part $\Omega_{\infty}$ of $\Omega$ given in Proposition 20, that is,

$$
\Omega_{\infty}=\bigcup_{i=1}^{h_{Q}} \xi_{i} \Omega_{i, \infty}
$$

Let $\Omega_{\infty}^{\circ}$ and $\Omega_{\infty}^{-}$be the interior and the closure of $\Omega_{\infty}$ in $G\left(\mathrm{k}_{\infty}\right)^{1}$, respectively. The projection from $G(\mathbb{A})^{1}=G(\mathrm{k}) G_{\mathrm{A}, \infty}^{1}$ to the infinite component $G\left(\mathrm{k}_{\infty}\right)^{1}$ gives an isomorphism $G(\mathrm{k}) \backslash G(\mathbb{A})^{1} / K_{f} \cong G_{\circ} \backslash G\left(\mathrm{k}_{\infty}\right)^{1}$. Since $\Omega$ is a fundamental domain of $G(\mathbb{A})^{1}$ with respect to $G(\mathrm{k})$ by Theorem 15 , we have $G_{\circ} \Omega_{\infty}^{-}=G\left(\mathrm{k}_{\infty}\right)^{1}$.

Corollary 22. If $h_{Q}=1$, then $\Omega_{\infty}$ is a fundamental domain of $G\left(\mathrm{k}_{\infty}\right)^{1}$ with respect to $G_{\mathrm{o}}$.

Proof. Since $\Omega_{\infty}=\Omega_{1, \infty}$ is a relatively open set in $\mathrm{R}_{e, \infty}^{*}$, we have $\Omega_{\infty}^{\circ}=$ $\Omega_{\infty} \cap \mathrm{R}_{e, \infty}^{\circ}$. Thus the closure of $\Omega_{\infty}^{\circ}$ coincides with $\Omega_{\infty}^{-}$. If $\Omega_{\infty}^{\circ} \cap g \Omega_{\infty}^{-} \neq \varnothing$ for $g \in G_{\mathrm{o}}$, then $\left(\Omega_{\infty}^{\circ} \times K_{f}\right) \cap g\left(\Omega_{\infty}^{-} \times K_{f}\right) \neq \varnothing$ because $g K_{f}=K_{f}$. This implies $g=e$ since $\Omega_{\infty}^{\circ} \times K_{f}$ is an open fundamental domain of $G(\mathbb{A})^{1}$ with respect to $G(\mathrm{k})$.

\section{Examples}

Example 3. Let $G$ be a general linear group $\mathrm{GL}_{n}$ defined over $\mathbb{Q}$. We continue an illustration given in Examples 1 and 2. We fix an integer $k \in\{1, \ldots, n-1\}$, and 
let

$$
Q(\mathbb{Q})=\left\{\left(\begin{array}{ll}
a & b \\
0 & d
\end{array}\right): a \in \mathrm{GL}_{k}(\mathbb{Q}), b \in \mathrm{M}_{k, n-k}(\mathbb{Q}), d \in \mathrm{GL}_{n-k}(\mathbb{Q})\right\} .
$$

Since $h_{Q}=1$, we have $\xi_{1}=e$ and $Q_{1}=Q$.

Let $\mathrm{P}_{n}$ be the cone of positive definite $n$ by $n$ real symmetric matrices, and let $\mathrm{P}_{n}^{1}$ be the intersection of $\mathrm{P}_{n}$ and $\mathrm{SL}_{n}(\mathbb{R})$. The group $G\left(\mathbb{Q}_{\infty}\right)=\mathrm{GL}_{n}(\mathbb{R})$ acts on $\mathrm{P}_{n}$ from the right by $(A, g) \mapsto A[g]={ }^{t} g A g$ for $(A, g) \in \mathrm{P}_{n} \times G\left(\mathbb{Q}_{\infty}\right)$. The maximal compact subgroup $K_{\infty}$ of $G\left(\mathbb{Q}_{\infty}\right)$, defined as in Example 2, stabilizes the identity matrix $I_{n} \in \mathrm{P}_{n}$. The map $\pi: g \mapsto{ }^{t} g^{-1} g^{-1}$ from $G\left(\mathbb{Q}_{\infty}\right)$ onto $\mathrm{P}_{n}$ gives an isomorphism between $G\left(\mathbb{Q}_{\infty}\right) / K_{\infty}$ and $\mathrm{P}_{n}$. Since

$$
G\left(\mathbb{Q}_{\infty}\right)^{1}=\left\{g \in G\left(\mathbb{Q}_{\infty}\right): \operatorname{det} g= \pm 1\right\},
$$

we have $G\left(\mathbb{Q}_{\infty}\right)^{1} / K_{\infty} \cong \pi\left(G\left(\mathbb{Q}_{\infty}\right)^{1}\right)=\mathrm{P}_{n}^{1}$. An element $A \in \mathrm{P}_{n}$ is written as

$$
A=\left(\begin{array}{cc}
I_{k} & 0 \\
t_{u} & I_{n-k}
\end{array}\right)\left(\begin{array}{cc}
v & 0 \\
0 & w
\end{array}\right)\left(\begin{array}{cc}
I_{k} & u \\
0 & I_{n-k}
\end{array}\right),
$$

where $v \in \mathrm{P}_{k}, w \in \mathrm{P}_{n-k}$ and $u \in \mathrm{M}_{k, n-k}(\mathbb{R})$. We write $u_{A}, A^{[k]}$ and $A_{[n-k]}$ for $u$, $v$ and $w$, respectively.

By definition, $G_{\mathbb{Z}}=G(\mathbb{Q}) \cap G_{\mathbb{A}, \infty}$ and $Q_{\mathbb{Z}}=Q(\mathbb{Q}) \cap G_{\mathbb{A}, \infty}$ are just the groups $\mathrm{GL}_{n}(\mathbb{Z})$ and $Q(\mathbb{Q}) \cap \mathrm{GL}_{n}(\mathbb{Z})$ of unimodular integral matrices in $G(\mathbb{Q})$ and $Q(\mathbb{Q})$, respectively. As in Example 2, $X_{\gamma}$ stands for the $n$ by $k$ matrix consisting of the first $k$-columns of $\gamma \in G_{\mathbb{Z}}$, and $\mathrm{M}_{n, k}(\mathbb{Z})^{*}$ stands for the set of $X_{\gamma}$ for all $\gamma \in G_{\mathbb{Z}}$. We define the closed subset $\mathrm{F}_{n, k}$ of $\mathrm{P}_{n}$ as follows:

$$
\mathrm{F}_{n, k}=\left\{A \in \mathrm{P}_{n}: \operatorname{det} A^{[k]} \leq \operatorname{det}\left({ }^{t} X A X\right) \text { for all } X \in \mathrm{M}_{n, k}(\mathbb{Z})^{*}\right\} .
$$

In Example 2, we showed

$$
H_{Q}(\gamma g)=\operatorname{det}\left({ }^{t} X_{\gamma^{-1}} \pi(g) X_{\gamma^{-1}}\right)^{n / 2 r}
$$

for any $\gamma \in G_{\mathbb{Z}}$ and $g \in G\left(\mathbb{Q}_{\infty}\right)^{1}$. Since $H_{Q}(g)=\left(\operatorname{det} \pi(g)^{[k]}\right)^{n / 2 r}$, we obtain

$$
\mathrm{R}_{e, \infty} / K_{\infty} \cong \pi\left(\mathrm{R}_{e, \infty}\right)=\mathrm{F}_{n, k} \cap \mathrm{SL}_{n}(\mathbb{R}) .
$$

Therefore, $Q_{\mathbb{Z}} \backslash \mathrm{R}_{e, \infty} / K_{\infty}$ is isomorphic to $\left(\mathrm{F}_{n, k} \cap \mathrm{SL}_{n}(\mathbb{R})\right) / Q_{\mathbb{Z}}$. If $\gamma \in Q_{\mathbb{Z}}$ is of the form

$$
\gamma=\left(\begin{array}{ll}
a & b \\
0 & d
\end{array}\right)
$$

with $a \in \mathrm{GL}_{k}(\mathbb{Z}), d \in \mathrm{GL}_{n-k}(\mathbb{Z})$ and $b \in \mathrm{M}_{k, n-k}(\mathbb{Z})$, then components of ${ }^{t} \gamma A \gamma$ for $A \in \mathrm{P}_{n}$ are given by

$$
u_{t_{\gamma} A \gamma}=a^{-1}\left(u_{A} d+b\right), \quad\left({ }^{t} \gamma A \gamma\right)^{[k]}={ }^{t} a A^{[k]} a, \quad\left({ }^{t} \gamma A \gamma\right)_{[n-k]}={ }^{t} d A_{[n-k]} d .
$$


Let $\mathfrak{D}$ and $\mathfrak{E}$ be arbitrary fundamental domains for the quotients $\mathrm{P}_{k} / \mathrm{GL}_{k}(\mathbb{Z})$ and $\mathrm{P}_{n-k} / \mathrm{GL}_{n-k}(\mathbb{Z})$, respectively. We define the subset $\mathrm{F}_{n, k}(\mathfrak{D}, \mathfrak{E})$ of $\mathrm{F}_{n, k}$ as

$$
\begin{aligned}
\mathrm{F}_{n, k}(\mathfrak{D}, \mathfrak{E})=\left\{A \in \mathrm{F}_{n, k}: A^{[k]}\right. & \in \mathfrak{D}, A_{[n-k]} \in \mathfrak{E}, \\
u_{A} & \left.=\left(u_{i j}\right),-\frac{1}{2} \leq u_{i j} \leq \frac{1}{2} \text { for all } i, j, \text { and } 0 \leq u_{11}\right\} .
\end{aligned}
$$

Since $\mathrm{F}_{n, k}(\mathfrak{D}, \mathfrak{E})$ is a fundamental domain of $\mathrm{F}_{n, k}$ with respect to $Q_{\mathbb{Z}}$, the inverse image $\pi^{-1}\left(\mathrm{~F}_{n, k}(\mathfrak{D}, \mathfrak{E}) \cap \mathrm{SL}_{n}(\mathbb{R})\right)$ of $\mathrm{F}_{n, k}(\mathfrak{D}, \mathfrak{E}) \cap \mathrm{SL}_{n}(\mathbb{R})$ gives a fundamental domain of $\mathrm{R}_{e, \infty}$ with respect to $Q_{\mathbb{Z}}$. As a consequence of Theorem 15 and Proposition 20, the set

$$
\pi^{-1}\left(\mathrm{~F}_{n, k}(\mathfrak{D}, \mathfrak{E}) \cap \mathrm{SL}_{n}(\mathbb{R})\right) \times K_{f}
$$

gives a fundamental domain of $G(\mathbb{A})^{1}$ with respect to $G(\mathbb{Q})$. Moreover, from Corollary 22, it follows that $\mathrm{F}_{n, k}(\mathfrak{D}, \mathfrak{E})$ is a fundamental domain of $\mathrm{P}_{n}$ with respect to $\mathrm{GL}_{n}(\mathbb{Z})$.

In the case of $k=1$, this gives an inductive construction of a fundamental domain $\Omega_{n}$ of $\mathrm{P}_{n}$ with respect to $\mathrm{GL}_{n}(\mathbb{Z})$ as follows. First, put $\Omega_{2}=\mathrm{F}_{2,1}\left(\mathrm{P}_{1}, \mathrm{P}_{1}\right)$. By definition, $\Omega_{2}$ is Minkowski's fundamental domain of $P_{2}$. Then we define inductively $\Omega_{3}=F_{3,1}\left(P_{1}, \Omega_{2}\right), \ldots, \Omega_{n}=F_{n, 1}\left(P_{1}, \Omega_{n-1}\right)$. The domain $\Omega_{n}$ coincides with Grenier's fundamental domain [1988].

Finally, we show that, in the case of $k=1, \mathrm{R}_{e, \infty} / K_{\infty}$ corresponds to a face of the Ryshkov polyhedron $\mathrm{R}(\mathrm{m})=\left\{A \in \mathrm{P}_{n}: \mathrm{m}(A)=\min _{0 \neq x \in \mathbb{Z}^{n}}{ }^{t} x A x \geq 1\right\}$. For $A \in \mathrm{P}_{n}$, let $S(A)$ denote the set of minimal integral vectors of $A$ :

$$
S(A)=\left\{x \in \mathbb{Z}^{n}: \mathrm{m}(A)={ }^{t} x A x\right\} .
$$

We take $\boldsymbol{e}_{1}={ }^{t}(1,0, \ldots, 0) \in \mathbb{Z}^{n}$. It is obvious that the subset $\left\{A \in \mathrm{P}_{n}: \boldsymbol{e}_{1} \in\right.$ $S(A)\}$ of $\mathrm{P}_{n}$ coincides with $\mathrm{F}_{n, 1}$. As was shown in [Watanabe 2012, Lemma 1.5], $\mathscr{F}_{\left\{\boldsymbol{e}_{1}\right\}}=\mathrm{F}_{n, 1} \cap \partial \mathrm{R}(\mathrm{m})=\left\{A \in \mathrm{F}_{n, 1}: \mathrm{m}(A)=1\right\}$ is a face of $\mathrm{R}(\mathrm{m})$. It is easy to see that the map $A \mapsto \mathrm{m}(A)^{-1} A$ gives a bijection from $\mathrm{F}_{n, 1} \cap \mathrm{SL}_{n}(\mathbb{R})$ onto $\mathscr{F}_{\left\{e_{1}\right\}}$. Therefore, $\mathrm{R}_{e, \infty} / K_{\infty} \cong \pi\left(\mathrm{R}_{e, \infty}\right)$ corresponds to $\mathscr{F}_{\left\{\boldsymbol{e}_{1}\right\}}$.

Example 4. Let $\mathrm{k}$ be a totally real number field of degree $r$ and $n=2 m$ be an even integer. We consider a symplectic group

$$
G(\mathrm{k})=\mathrm{Sp}_{n}(\mathrm{k})=\left\{g \in \mathrm{GL}_{2 m}(\mathrm{k}):{ }^{t} g\left(\begin{array}{cc}
0 & -I_{m} \\
I_{m} & 0
\end{array}\right) g=\left(\begin{array}{cc}
0 & -I_{m} \\
I_{m} & 0
\end{array}\right)\right\} .
$$

For a fixed $k \in\{1,2, \ldots, m\}$, let $Q$ denote the maximal parabolic subgroup of $G$ given by

$$
Q(\mathrm{k})=U(\mathrm{k}) M(\mathrm{k})
$$


where

$$
\begin{aligned}
& M(\mathrm{k})=\left\{\delta(a, b)=\left(\begin{array}{cccc}
a & 0 & 0 & 0 \\
0 & b_{11} & 0 & b_{12} \\
0 & 0 & t^{-1} & 0 \\
0 & b_{21} & 0 & b_{22}
\end{array}\right): \quad \begin{array}{c}
a \in \mathrm{GL}_{k}(\mathrm{k}), \\
\left(b_{i j}\right) \in \mathrm{Sp}_{2(m-k)}(\mathrm{k})
\end{array}\right\}, \\
& U(\mathrm{k})=\left\{\left(\begin{array}{cccc}
I_{k} & * & * & * \\
0 & I_{m-k} & * & 0 \\
0 & 0 & I_{k} & 0 \\
0 & 0 & * & I_{m-k}
\end{array}\right) \in G(\mathrm{k})\right\} .
\end{aligned}
$$

The module of k-rational characters $X^{*}(M)_{\mathrm{k}}$ of $M$ is a free $\mathbb{Z}$-module of rank 1 generated by the character

$$
\widehat{\alpha}_{Q}(\delta(a, b))=\operatorname{det} a .
$$

The height $H_{Q}: G(\mathbb{A}) \rightarrow \mathbb{R}_{>0}$ is given by $H_{Q}(g)=|\operatorname{det} a|_{\mathbb{A}}^{-1}$ if $g=u \delta(a, b) h$ with $u \in U(\mathbb{A}), \delta(a, b) \in M(\mathbb{A})$ and $h \in K$.

We restrict ourselves to the case $k=m$. An element of $M(\mathbb{A})$ is denoted by

Let

$$
\delta(a)=\left(\begin{array}{cc}
a & 0 \\
0 & { }^{t} a^{-1}
\end{array}\right), \quad a \in \mathrm{GL}_{m}(\mathbb{A}) .
$$

$$
\mathrm{H}_{m}=\left\{Z \in \mathrm{M}_{m}(\mathbb{C}):{ }^{t} Z=Z, \operatorname{Im} Z \in \mathrm{P}_{m}\right\}
$$

be the Siegel upper half space and $\mathrm{H}_{m}^{r}$ the direct product of $r$ copies of $\mathrm{H}_{m}$. For $Z=\left(Z_{\sigma}\right)_{\sigma \in \mathrm{p}_{\infty}} \in \mathrm{H}_{m}^{r}, \operatorname{Re} Z, \operatorname{Im} Z$ and det $Z$ stand for $\left(\operatorname{Re} Z_{\sigma}\right)_{\sigma \in \mathrm{p}_{\infty}},\left(\operatorname{Im} Z_{\sigma}\right)_{\sigma \in \mathrm{p}_{\infty}}$ and $\left(\operatorname{det} Z_{\sigma}\right)_{\sigma \in \mathrm{p}_{\infty}}$, respectively. The group $G\left(\mathrm{k}_{\infty}\right)$ acts transitively on $\mathrm{H}_{m}^{r}$ by

$$
g\langle Z\rangle=\left(\left(a_{\sigma} Z_{\sigma}+b_{\sigma}\right)\left(c_{\sigma} Z_{\sigma}+d_{\sigma}\right)^{-1}\right)_{\sigma \in \mathrm{p}_{\infty}}
$$

for $Z=\left(Z_{\sigma}\right) \in \mathrm{H}_{m}^{r}$ and

$$
g=\left(g_{\sigma}\right)=\left(\begin{array}{ll}
a_{\sigma} & b_{\sigma} \\
c_{\sigma} & d_{\sigma}
\end{array}\right)_{\sigma \in \mathrm{p}_{\infty}} \in G\left(\mathrm{k}_{\infty}\right) .
$$

The stabilizer $K_{\infty}$ of $Z_{0}=\left(\sqrt{-1} I_{m}, \ldots, \sqrt{-1} I_{m}\right) \in \mathrm{H}_{m}^{r}$ in $G\left(\mathrm{k}_{\infty}\right)$ is a maximal compact subgroup of $G\left(\mathrm{k}_{\infty}\right)$. We choose $K$ as $K_{\infty} \times \prod_{\sigma \in \mathrm{p}_{f}} \mathrm{Sp}_{n}\left(\mathrm{o}_{\sigma}\right)$. The map $\pi: g_{\infty} \mapsto g\left\langle Z_{0}\right\rangle$ from $G\left(\mathrm{k}_{\infty}\right)$ onto $\mathrm{H}_{m}^{r}$ gives an isomorphism $G\left(\mathrm{k}_{\infty}\right) / K_{\infty} \cong \mathrm{H}_{m}^{r}$, and hence $G(\mathrm{k}) \backslash G(\mathbb{A}) / K \cong G_{\mathrm{o}} \backslash \mathrm{H}_{m}^{r}$. Since $\operatorname{Im}\left\{(u \delta(a) h)\left\langle Z_{0}\right\rangle\right\}=a^{t} a$ holds for $u \in U\left(\mathrm{k}_{\infty}\right), a \in \mathrm{GL}_{m}\left(\mathrm{k}_{\infty}\right)$ and $h \in K_{\infty}$, we have

$$
H_{Q}\left(g_{\infty}\right)=\operatorname{Nr}_{\mathrm{k}_{\infty} / \mathbb{R}}\left(\operatorname{det} \operatorname{Im}\left\{g_{\infty}\left\langle Z_{0}\right\rangle\right\}\right)^{-1 / 2}=\left(\prod_{\sigma \in \mathrm{p}_{\infty}} \operatorname{det} \operatorname{Im}\left\{g_{\sigma}\left(\sqrt{-1} I_{m}\right)\right\}\right)^{-1 / 2}
$$

for any $g_{\infty}=\left(g_{\sigma}\right) \in G\left(\mathrm{k}_{\infty}\right)$, where $\mathrm{Nr}_{\mathrm{k}_{\infty} / \mathbb{R}}$ denotes the norm of $\mathrm{k}_{\infty}$ over $\mathbb{R}$. 
The class number $h_{Q}$ of $M \cong \mathrm{GL}_{m}$ defined over $\mathrm{k}$ is equal to the class number $h_{\mathrm{k}}$ of $\mathrm{k}$. We assume $h_{\mathrm{k}}=1$ for simplicity. Then $G(\mathrm{k})=Q(\mathrm{k}) G_{\mathrm{o}}$ and $G(\mathbb{A})=$ $Q(\mathrm{k}) G_{\mathrm{A}, \infty}$, and hence

$$
\mathrm{m}_{Q}\left(g_{\infty}\right)=\min _{\gamma \in G_{\circ}} H_{Q}\left(\gamma g_{\infty}\right)
$$

Since

$$
\mathrm{Nr}_{\mathrm{k}_{\infty} / \mathbb{R}}(\operatorname{det} \operatorname{Im}\{\gamma\langle Z\rangle\})=\prod_{\sigma \in \mathrm{p}_{\infty}}\left|\operatorname{det}\left(\sigma(c) Z_{\sigma}+\sigma(d)\right)\right|^{-2} \mathrm{Nr}_{\mathrm{k}_{\infty} / \mathbb{R}}(\operatorname{det} \operatorname{Im} Z)
$$

for $Z=\left(Z_{\sigma}\right) \in \mathrm{H}_{m}^{r}$ and

$$
\gamma=\left(\begin{array}{ll}
* & * \\
c & d
\end{array}\right) \in G_{\mathrm{o}}=\operatorname{Sp}_{n}(\mathrm{o})
$$

the condition $\mathrm{m}_{Q}\left(g_{\infty}\right)=H_{Q}\left(g_{\infty}\right)$ of $g_{\infty}$ is equivalent with the following condition of $Z=g_{\infty}\left\langle Z_{0}\right\rangle$ :

$$
\prod_{\sigma \in \mathrm{p}_{\infty}}\left|\operatorname{det}\left(\sigma(c) Z_{\sigma}+\sigma(d)\right)\right| \geq 1 \quad \text { for all } \quad\left(\begin{array}{ll}
* & * \\
c & d
\end{array}\right) \in G_{\circ} .
$$

Therefore, the domain $R_{e, \infty}$ modulo $K_{\infty}$ is isomorphic to

$$
\mathrm{F}=\left\{\left(Z_{\sigma}\right) \in \mathrm{H}_{m}^{r}: \prod_{\sigma \in \mathrm{p}_{\infty}}\left|\operatorname{det}\left(\sigma(c) Z_{\sigma}+\sigma(d)\right)\right| \geq 1 \text { for all }\left(\begin{array}{ll}
* & * \\
c & d
\end{array}\right) \in G_{\circ}\right\} .
$$

Let $\mathfrak{C}$ be an arbitrary fundamental domain of the additive group $\mathrm{M}_{m}\left(\mathrm{k}_{\infty}\right)$ with respect to $\mathrm{M}_{m}(\mathrm{o})$, and let $\mathfrak{D}$ be an arbitrary fundamental domain of $\mathrm{P}_{m}^{r}$ with respect to $\mathrm{GL}_{m}(\mathrm{o})$. It is easy to see that

$$
\mathrm{F}(\mathfrak{C}, \mathfrak{D})=\{Z \in \mathrm{F}: \operatorname{Re} Z \in \mathfrak{C}, \operatorname{Im} Z \in \mathfrak{D}\}
$$

is a fundamental domain of $\mathrm{F}$ with respect to $Q_{\mathrm{o}}$. By Corollary $22, \mathrm{~F}(\mathfrak{C}, \mathfrak{D})$ is a fundamental domain of $\mathrm{H}_{m}^{r}$ with respect to $G_{\mathrm{o}}$.

If $k=\mathbb{Q}$ and $\mathfrak{D}$ is Minkowski's fundamental domain, then $F(\mathfrak{C}, \mathfrak{D})$ coincides with Siegel's fundamental domain [1939].

Acknowledgments. The author would like to thank Professor Takahiro Hayata for useful discussions.

\section{References}

[Baer and Levi 1931] R. Baer and F. Levi, "Stetige Funktionen in topologischen Räumen”, Math. Z. 34:1 (1931), 110-130. MR 1545244 Zbl 0002.16002 JFM 57.0731.02

[Bombieri and Gubler 2006] E. Bombieri and W. Gubler, Heights in Diophantine geometry, New Mathematical Monographs 4, Cambridge University Press, 2006. MR 2007a:11092 Zbl 1115.11034 [Borel 1963] A. Borel, "Some finiteness properties of adele groups over number fields", Inst. Hautes Études Sci. Publ. Math. 16 (1963), 5-30. MR 34 \#2578 Zbl 0135.08902 
[Borel 1991] A. Borel, Linear algebraic groups, 2nd ed., Graduate Texts in Mathematics 126, Springer, New York, 1991. MR 92d:20001 Zbl 0726.20030

[Borel and Tits 1965] A. Borel and J. Tits, "Groupes réductifs", Inst. Hautes Études Sci. Publ. Math. 27 (1965), 55-150. MR 34 \#7527 Zbl 0145.17402

[Grenier 1988] D. Grenier, "Fundamental domains for the general linear group", Pacific J. Math. 132:2 (1988), 293-317. MR 89d:11055 Zbl 0699.10045

[Humphreys 1990] J. E. Humphreys, Reflection groups and Coxeter groups, Cambridge Studies in Advanced Mathematics 29, Cambridge University Press, 1990. MR 92h:20002 Zbl 0725.20028

[Martinet 2003] J. Martinet, Perfect lattices in Euclidean spaces, Grundlehren der Mathematischen Wissenschaften 327, Springer, Berlin, 2003. MR 2003m:11099 Zbl 1017.11031

[Ryshkov 1970] S. S. Ryshkov, "The polyhedron $\mu(m)$ and some extremal problems in the geometry of numbers", Dokl. Akad. Nauk SSSR 194 (1970), 514-517. In Russian; translated in Sov. Math. Dokl. 11 (1970), 1240-1244. MR 43 \#2613 Zbl 0229.10013

[Schürmann 2009] A. Schürmann, Computational geometry of positive definite quadratic forms: polyhedral reduction theories, algorithms, and applications, University Lecture Series 48, Amer. Math. Soc., Providence, RI, 2009. MR 2010a:11130 Zbl 1185.52016

[Shimura 1994] G. Shimura, "Fractional and trigonometric expressions for matrices", Amer. Math. Monthly 101:8 (1994), 744-758. MR 96e:15053 Zbl 0836.15004

[Siegel 1939] C. L. Siegel, "Einführung in die Theorie der Modulfunktionen $n$-ten Grades", Math. Ann. 116 (1939), 617-657. MR 1,203f Zbl 0021.20302

[Terras 1988] A. Terras, Harmonic analysis on symmetric spaces and applications, II, Springer, Berlin, 1988. MR 89k:22017 Zbl 0668.10033

[Voronoï 1908] G. Voronoï, "Nouvelles applications des paramètres continus à la théorie des formes quadratiques, premier mémoire: sur quelques propriétés des formes quadratiques positives parfaites", J. Reine Angew. Math. 133 (1908), 97-178. JFM 38.0261.01

[van der Waerden 1935] B. L. van der Waerden, Gruppen von linearen Transformationen, Ergebnisse der Mathematik und ihrer Grenzgebiete 4:2, Springer, Berlin, 1935. Zbl 0011.10102 JFM 61.0105.03

[Watanabe 2000] T. Watanabe, “On an analog of Hermite's constant”, J. Lie Theory 10:1 (2000), 33-52. MR 2001a:11111 Zbl 1029.11031

[Watanabe 2003] T. Watanabe, "Fundamental Hermite constants of linear algebraic groups", J. Math. Soc. Japan 55:4 (2003), 1061-1080. MR 2005f:11260 Zbl 1103.11033

[Watanabe 2012] T. Watanabe, “A survey on Voronoï's theorem”, pp. 334-377 in Geometry and analysis of automorphic forms of several variables (Tokyo, 2009), edited by Y. Hamahata et al., Ser. Number Theory Appl. 7, World Scientific, Hackensack, NJ, 2012. MR 2908043 Zbl 1268.11090

Received March 29, 2013. Revised July 30, 2013.

TAKAO WATANABE

GRADUATE SCHOOL OF SCIENCE

OSAKA UNIVERSITY

MACHIKANEYAMA 1-1

TOYONAKA 560-0043

JAPAN

twatanabe@math.sci.osaka-u.ac.jp 


\title{
PACIFIC JOURNAL OF MATHEMATICS
}

\author{
msp.org/pjm
}

Founded in 1951 by E. F. Beckenbach (1906-1982) and F. Wolf (1904-1989)

\section{EDITORS}

Don Blasius (Managing Editor)

Department of Mathematics

University of California

Los Angeles, CA 90095-1555

blasius@math.ucla.edu

\author{
Paul Balmer \\ Department of Mathematics \\ University of California \\ Los Angeles, CA 90095-1555 \\ balmer@math.ucla.edu \\ Robert Finn \\ Department of Mathematics \\ Stanford University \\ Stanford, CA 94305-2125 \\ finn@math.stanford.edu \\ Sorin Popa \\ Department of Mathematics \\ University of California \\ Los Angeles, CA 90095-1555 \\ popa@math.ucla.edu
}

\author{
Vyjayanthi Chari \\ Department of Mathematics \\ University of California \\ Riverside, CA 92521-0135 \\ chari@math.ucr.edu \\ Kefeng Liu \\ Department of Mathematics \\ University of California \\ Los Angeles, CA 90095-1555 \\ liu@math.ucla.edu \\ Jie Qing \\ Department of Mathematics \\ University of California \\ Santa Cruz, CA 95064 \\ qing@ cats.ucsc.edu
}

\section{PRODUCTION}

Silvio Levy, Scientific Editor, production@msp.org

\section{SUPPORTING INSTITUTIONS}

ACADEMIA SINICA, TAIPEI

CALIFORNIA INST. OF TECHNOLOGY

INST. DE MATEMÁTICA PURA E APLICADA

KEIO UNIVERSITY

MATH. SCIENCES RESEARCH INSTITUTE

NEW MEXICO STATE UNIV.

OREGON STATE UNIV.

\author{
STANFORD UNIVERSITY \\ UNIV. OF BRITISH COLUMBIA \\ UNIV. OF CALIFORNIA, BERKELEY \\ UNIV. OF CALIFORNIA, DAVIS \\ UNIV. OF CALIFORNIA, LOS ANGELES \\ UNIV. OF CALIFORNIA, RIVERSIDE \\ UNIV. OF CALIFORNIA, SAN DIEGO \\ UNIV. OF CALIF., SANTA BARBARA
}

\author{
Daryl Cooper \\ Department of Mathematics \\ University of California \\ Santa Barbara, CA 93106-3080 \\ cooper@math.ucsb.edu \\ Jiang-Hua Lu \\ Department of Mathematics \\ The University of Hong Kong \\ Pokfulam Rd., Hong Kong \\ jhlu@maths.hku.hk \\ Paul Yang \\ Department of Mathematics \\ Princeton University \\ Princeton NJ 08544-1000 \\ yang@math.princeton.edu
}

These supporting institutions contribute to the cost of publication of this Journal, but they are not owners or publishers and have no responsibility for its contents or policies.

See inside back cover or msp.org/pjm for submission instructions.

The subscription price for 2014 is US $\$ 410 /$ year for the electronic version, and \$535/year for print and electronic.

Subscriptions, requests for back issues and changes of subscribers address should be sent to Pacific Journal of Mathematics, P.O. Box 4163, Berkeley, CA 94704-0163, U.S.A. The Pacific Journal of Mathematics is indexed by Mathematical Reviews, Zentralblatt MATH, PASCAL CNRS Index, Referativnyi Zhurnal, Current Mathematical Publications and Web of Knowledge (Science Citation Index).

The Pacific Journal of Mathematics (ISSN 0030-8730) at the University of California, c/o Department of Mathematics, 798 Evans Hall \#3840, Berkeley, CA 94720-3840, is published twelve times a year. Periodical rate postage paid at Berkeley, CA 94704, and additional mailing offices. POSTMASTER: send address changes to Pacific Journal of Mathematics, P.O. Box 4163, Berkeley, CA 94704-0163.

PJM peer review and production are managed by EditFLOW ${ }^{\circledR}$ from Mathematical Sciences Publishers.

\section{PUBLISHED BY}

\section{mathematical sciences publishers \\ nonprofit scientific publishing}

http://msp.org/

(C) 2014 Mathematical Sciences Publishers 


\section{PACIFIC JOURNAL OF MATHEMATICS}

Volume $270 \quad$ No. $1 \quad$ July 2014

Hermitian categories, extension of scalars and systems of sesquilinear forms

EVA BAYER-FLUCKIGER, URIYA A. FIRST and DANIEL A.

MOLDOVAN

Realizations of the three-point Lie algebra $\mathfrak{s l}(2, \mathscr{R}) \oplus\left(\Omega_{\mathscr{R}} / d \mathscr{R}\right)$

BEN COX and ELIZABETH JURISICH

Multi-bump bound state solutions for the quasilinear Schrödinger equation with critical frequency

YUXIA GUO and ZHONGWEI TANG

On stable solutions of the biharmonic problem with polynomial growth

Hatem Hajlaoui, Abdellaziz Harrabi and Dong Ye

Valuative multiplier ideals

ZHENGYU HU

Quasiconformal conjugacy classes of parabolic isometries of complex hyperbolic space

YOUNGJU KIM

On the distributional Hessian of the distance function

Carlo MantegazZa, Giovanni Mascellani and Gennady URALTSEV

Noether's problem for abelian extensions of cyclic $p$-groups

IVO M. MICHAILOV

Legendrian $\theta$-graphs

Danielle O’Donnol and Elena PaVelescu

A class of Neumann problems arising in conformal geometry

WEIMIN SHENG and LI-XIA YUAN

Ryshkov domains of reductive algebraic groups

TAKAO WATANABE 